\title{
A benchmark study of numerical implementations of the sea-level equation in GIA modelling
}

Martinec, Z.; Klemann, V.; van der Wal, W.; Riva, R. E. M; Spada, G.; Sun, Y.; Melini, D.; Kachuck, S. B.; Barletta, V.; Simon, K.

Total number of authors:

12

Published in:

Geophysical Journal International

Link to article, DOI:

$10.1093 / g j i / g g y 280$

Publication date:

2018

Document Version

Publisher's PDF, also known as Version of record

Link back to DTU Orbit

Citation (APA):

Martinec, Z., Klemann, V., van der Wal, W., Riva, R. E. M., Spada, G., Sun, Y., Melini, D., Kachuck, S. B., Barletta, V., Simon, K., A, G., \& James, T. (2018). A benchmark study of numerical implementations of the sealevel equation in GIA modelling. Geophysical Journal International, 215(1), 389-414.

https://doi.org/10.1093/gji/ggy280

\section{General rights}

Copyright and moral rights for the publications made accessible in the public portal are retained by the authors and/or other copyright owners and it is a condition of accessing publications that users recognise and abide by the legal requirements associated with these rights.

- Users may download and print one copy of any publication from the public portal for the purpose of private study or research.

- You may not further distribute the material or use it for any profit-making activity or commercial gain

- You may freely distribute the URL identifying the publication in the public portal 


\title{
A benchmark study of numerical implementations of the sea level equation in GIA modelling
}

\author{
Z. Martinec, ${ }^{1,2}$ V. Klemann, ${ }^{3}$ W. van der Wal, ${ }^{4}$ R. E. M. Riva, ${ }^{5}$ G. Spada, ${ }^{6}$ Y. Sun, ${ }^{5}$ \\ D. Melini, ${ }^{7}$ S. B. Kachuck, ${ }^{8}$ V. Barletta, ${ }^{9}$ K. Simon, ${ }^{5}$ G. $A^{10}$ and T. S. James ${ }^{11}$ \\ ${ }^{1}$ Dublin Institute for Advanced Studies, 5 Merrion Square, D02 Y006 Dublin 2, Ireland. E-mail: zdenek@cp.dias.ie \\ ${ }^{2}$ Department of Geophysics, Faculty of Mathematics and Physics, Charles University, V Holešovičkách 2, 18000 Prague 8, Czech Republic \\ ${ }^{3}$ Helmholtz Centre Potsdam, GFZ German Research Centre for Geosciences, Section 1.3, Earth System Modelling, 14473 Potsdam, Germany \\ ${ }^{4}$ TU Delft, Faculty of Aerospace Engineering, 2629 HS Delft, The Netherlands \\ ${ }^{5}$ TU Delft, Faculty of Civil Engineering and Geosciences \& TU Delft Climate Institute, 2628 CN Delft, The Netherlands \\ ${ }^{6}$ Dipartimento di Scienze Pure e Applicate, Università di Urbino 'Carlo Bo', 61029 Urbino, Italy \\ ${ }^{7}$ Istituto Nazionale di Geofisica e Vulcanologia, 00143 Rome, Italy \\ ${ }^{8}$ Department of Physics, Cornell University, Ithaca, NY 14853, USA \\ ${ }^{9}$ DTU Space, Geodynamics, DK-2800 Kgs. Lyngby, Denmark \\ ${ }^{10}$ Department of Earth System Science, University of California, Irvine, CA 92697, USA \\ ${ }^{11}$ Geological Survey of Canada - Pacific Division, Sidney, British Columbia V8L 4B2, Canada
}

Accepted 2018 July 12. Received 2018 February 20; in original form 2018 July 07

\begin{abstract}
SUMMAR Y
The ocean load in glacial isostatic adjustment (GIA) modelling is represented by the so-called sea level equation (SLE). The SLE describes the mass redistribution of water between ice sheets and oceans on a deforming Earth. Despite various teams independently investigating GIA, there has been no systematic intercomparison among the numerical solvers of the SLE through which the methods may be validated. The goal of this paper is to present a series of synthetic examples designed for testing and comparing the numerical implementations of the SLE in GIA modelling. The 10 numerical codes tested combine various temporal and spatial parametrizations. The time-domain or Laplace-domain discretizations are used to solve the SLE through time, while spherical harmonics, finite differences or finite elements parametrize the GIA-related field variables spatially. The surface ice-water load and solid Earth's topography are represented spatially either on an equiangular grid, a Gauss-Legendre or an equiarea grid with icosahedron-shaped spherical pixels. Comparisons are made in a series of five benchmark examples with an increasing degree of complexity. Due to the complexity of the SLE, there is no analytical solution to it. The accuracy of the numerical implementations is therefore assessed by the differences of the individual solutions with respect to a reference solution. While the benchmark study does not result in GIA predictions for a realistic loading scenario, we establish a set of agreed-upon results that can be extended in the future by including more complex case studies, such as solutions with realistic loading scenarios, the rotational feedback in the linear-momentum equation, and by considering a 3-D viscosity structure of the Earth's mantle. The test computations performed so far show very good agreement between the individual results and their ability to capture the main features of sea-surface variation and the surface vertical displacement. The differences found can often be attributed to the different approximations inherent in the various algorithms. This shows the accuracy that can be expected from different implementations of the SLE, which helps to assess differences noted in the literature between predictions for realistic loading cases.
\end{abstract}

Key words: Sea level change; Numerical solutions; Dynamics: gravity and tectonics; Dynamics of lithosphere and mantle; Mechanics, theory, and modelling; Rheology: mantle.

\section{INTRODUCTION}

The redistribution of ice and water over the Earth's surface during glacial cycles due to changes in climate induces 3-D crustal motion, gravity field variations, changes in sea level and perturbations in the rotation of the Earth, all of which are known collectively as glacial 
isostatic adjustment (GIA). The forward modelling of the viscoelastic response of the Earth to a surface load is well established for a linear Maxwell rheology and several numerical methods have been proposed. The viscoelastic normal mode (VNM) method (e.g. Peltier 1974; Wu \& Peltier 1982), the finite element method (e.g. Gasperini \& Sabadini 1989; Kaufmann \& Wu 1998; Wu 2004; Steffen et al. 2006), the finite volume method (Latychev et al. 2005) and the spectral finite element method (Martinec 2000) are those mostly used. They differ in terms of the parametrization of time (time-domain versus Laplace-domain) and space (finite elements, finite differences, spherical harmonics). The GIA community previously collaborated on a benchmark study (Spada et al. 2011), which provided a systematic intercomparison of GIA numerical codes for a suite of several benchmarks. These activities were undertaken within the framework of the European COST Action ES0701 'Improved constraints on models of Glacial Isostatic Adjustment' ${ }^{1}$. The current benchmark study is a follow-on to the Spada et al. (2011) study. Here, the comparison is made for the case of surface-mass loading prescribed by the sea level equation (SLE).

The mathematical description of a realistic ocean load in GIA modelling has been under active and extensive development during the last decades (e.g. Peltier 1974, 1998; Farrell \& Clark 1976; Nakada \& Lambeck 1987; Mitrovica \& Peltier 1991; Mitrovica 1994; Milne et al. 1999; Lambeck et al. 2003; Kendall et al. 2005; Hagedoorn et al. 2007) and the theory of the SLE is now well established. The original form of the SLE by Farrell \& Clark (1976) has been extended for a time-varying ocean geometry (Lambeck \& Nakada 1990), floating ice in shallow basins (Lambeck et al. 2003; Milne \& Mitrovica 1998) and the Earth-rotation term in the gravitational forcing (Han \& Wahr 1989; Milne \& Mitrovica 1998).

There has been, however, no dedicated benchmark study focusing on the implementation of the SLE in GIA numerical codes. The purpose of this study is therefore to fill this gap by designing suitable synthetic examples that implement the SLE and compare the performance of different GIA numerical codes. There are several motivations for this benchmark study, which are similar to those introduced in Spada et al. (2011). First, it is currently unknown what the uncertainty is that results from differences in implementing the SLE, which can be significant (Barletta \& Bordoni 2013). Second, because details in the implementation of the SLE are sometimes omitted in publications, it is difficult to benchmark a solution of the SLE. An example of such difficulties can be seen in the case where a published prediction of sea level change (see the discussion in Mitrovica et al. 2011) resulted in large uncertainties in the accepted sea level rise presented in a report to the Dutch government (Vellinga et al. 2008).

The assumptions we have made in this paper include:

(1) The coordinate system coincides with the centre-of-mass frame (e.g. Klemann \& Martinec 2011).

(2) The earth model is incompressible and non-rotating.

(3) Ocean circulation is neglected; hence, the ocean surface remains an equipotential surface throughout time.

(4) The exchange of surface load between the ice sheets and the ocean basins is immediate, meaning that water does not remain on the land surface or in the atmosphere.

(5) Ice and ocean-water density are constant $\left(\varrho_{\text {ice }}=931 \mathrm{~kg} \mathrm{~m}^{-3}\right.$ and $\left.\varrho_{\mathrm{w}}=1000 \mathrm{~kg} \mathrm{~m}^{-3}\right)$.

The outline of this paper is as follows. The next section presents the theoretical background of the SLE. This is followed by a description of the five employed benchmark exercises and ten considered numerical codes. The results of the benchmark exercises are then presented and discussed. The main results of our study are summarized in the conclusion.

\section{MATHEMATICAL MODEL OF THE SLE}

When dealing with sea level change, it is useful to introduce two important quantities, the ocean function and relative sea level height. The ocean function $\mathcal{O}$ represents the surface distribution of oceans and continents; it is equal to 1 on the oceans and 0 on the continents. Since the geometry of the oceans varies over time, $\mathcal{O}=\mathcal{O}(\Omega, t)$, where $\Omega=(\vartheta, \lambda)$ denotes a pair of angular spherical coordinates, that is, spherical co-latitude $\vartheta$ and longitude $\lambda$. In particular, the ocean function at an initial time $t_{0}$ is defined by

$\mathcal{O}^{(0)}(\Omega)=\left\{\begin{array}{l}1 \text { if } \zeta^{(0)}(\Omega)<0, \\ 0 \text { otherwise, }\end{array}\right.$

where $\zeta^{(0)}(\Omega)$ represents the solid Earth's topography (SET) at $t_{0}$. We will assume that the Earth is in isostatic equilibrium at $t_{0}$. For $t>t_{0}$, the Earth's surface is loaded by ice and water with the area density $\sigma$ of the form

$\sigma(\Omega, t)=\sigma_{\text {ice }}(\Omega, t)+\varrho_{\mathrm{w}} s(\Omega, t)$,

where $\sigma_{\text {ice }}(\Omega, t)$ and $\varrho_{\mathrm{w}} s(\Omega, t)$ are spatially- and temporally-varying continental ice load (defined by eq. (28)) and ocean water load, respectively, relative to the ice and water distributions at $t_{0}$. The ice loading history $\sigma_{\text {ice }}(\Omega, t)$ is considered as a prescribed input parameter for the present benchmark study. The ocean load is proportional to the relative sea level (RSL) height $s(\Omega, t)$, which is defined as the sea level height at time $t$ relative to the sea level height at $t_{0}$,

$s(\Omega, t)=h_{\mathrm{w}}(\Omega, t)-h_{\mathrm{w}}\left(\Omega, t_{0}\right)$.

\footnotetext{
${ }^{1}$ http://www.cost-es0701.gcparks.com/
} 
where the sea level height $h_{\mathrm{w}}(\Omega, t)$ is the radial distance from the ocean bottom to the sea surface at the point $\Omega$ and time $t$. In addition to $s(\Omega$, $t$ ), we define the sea-surface variation $S(\Omega, t)$ as the radial distance of the sea surface at time $t$ relative to the radial distance of the sea surface at time $t_{0}$,

$S(\Omega, t)=r_{\mathrm{w}}(\Omega, t)-r_{\mathrm{w}}\left(\Omega, t_{0}\right)$,

where $r_{\mathrm{w}}(\Omega, t)$ is the distance from the geocentre to the sea surface at the point $\Omega$ and time $t$. Denoting the distance of the ocean bottom from the geocentre by $r_{\mathrm{f}}(\Omega, t)$, we have $s(\Omega, t)=r_{\mathrm{w}}(\Omega, t)-r_{\mathrm{f}}(\Omega, t)-\left[r_{\mathrm{w}}\left(\Omega, t_{0}\right)-r_{\mathrm{f}}\left(\Omega, t_{0}\right)\right]$, or

$s(\Omega, t)=S(\Omega, t)-U(\Omega, t)$,

where $U(\Omega, t)$ is the vertical displacement of the ocean bottom at the point $\Omega$ and time $t$. In particular, $s(\Omega, t)=S(\Omega, t)$ for a rigid earth model.

The RSL height $s(\Omega, t)$ is determined by the SLE, which yields the gravitationally consistent spatio-temporal evolution of sea level following the deformation response of the solid Earth and the redistribution of ocean-water masses according to continental ice mass loss and coastline migration. The SLE can be expressed in the form (Hagedoorn et al. 2007)

$s(\Omega, t)=h_{\mathrm{UF}}(t) \mathcal{O}(\Omega, t)+s_{\mathrm{NU}}(\Omega, t)$,

where $h_{\mathrm{UF}}$ is the thickness of the uniform water layer and $s_{\mathrm{NU}}$ is the non-uniform contribution to the RSL height. The respective contributions $h_{\mathrm{UF}}$ and $s_{\mathrm{NU}}$ are given by two principles applied at any time $t>t_{0}$ : (i) mass is conserved in the ice-ocean system, that is, accumulated ice is extracted from the ocean and melting ice returns to the ocean; (ii) the sea surface is an equipotential surface of gravitational potential. The respective principles will be referred to as the principle of mass conservation and the principle of equipotential surface, respectively. In the following, we present the specific situations of ocean geometry that will be used in the benchmark exercises.

\subsection{Fixed ocean geometry}

We will apply the two principles for various ice-ocean water distributions. Let us begin with the case where the ocean geometry is prescribed a priori at time $t_{0}$ and is fixed throughout time, that is, $\mathcal{O}(\Omega, t)=\mathcal{O}^{(0)}(\Omega)$. We further assume that ice and ocean water never overlap, which means that the ice load never extends to the ocean domain. Mathematically,

$\sigma_{\text {ice }}(\Omega, t)=\sigma_{\text {ice }}(\Omega, t)\left[1-\mathcal{O}^{(0)}(\Omega)\right]$.

In view of the principle of equipotential surface, the non-uniform contribution to the RSL height is

$s_{\mathrm{NU}}(\Omega, t)=[N(\Omega, t)-U(\Omega, t)] \mathcal{O}^{(0)}(\Omega)$,

where

$N(\Omega, t)=-\frac{\Phi(\Omega, t)}{g_{0}}$

is the geoid displacement. Here, $\Phi$ and $g_{0}$ are the gravitational potential increment and unperturbed gravitation on the Earth's surface, respectively. Eq. (8) along with eq. (6) says that the RSL height is reckoned from the ocean bottom displaced by $U$ to the sea surface displaced by $h_{\mathrm{UF}}+N$.

The principle of mass conservation, that is, the constraint

$\int_{\Omega_{0}} \sigma(\Omega, t) \mathrm{d} \Omega=0$

where $\Omega_{0}$ is the full solid angle and $\mathrm{d} \Omega$ is its infinitesimal element, now takes the form

$\int_{\Omega_{0}} \sigma_{\text {ice }}(\Omega, t)\left[1-\mathcal{O}^{(0)}(\Omega)\right] \mathrm{d} \Omega+\varrho_{\mathrm{w}} h_{\mathrm{UF}}(t) \int_{\Omega_{0}} \mathcal{O}^{(0)}(\Omega) \mathrm{d} \Omega+\varrho_{\mathrm{w}} \int_{\Omega_{0}}[N(\Omega, t)-U(\Omega, t)] \mathcal{O}^{(0)}(\Omega) \mathrm{d} \Omega=0$.

This gives $h_{\mathrm{UF}}$ as

$h_{\mathrm{UF}}(t)=-\frac{1}{\overline{\mathcal{O}^{(0)}}}\left[\frac{1}{\varrho_{\mathrm{W}}} \overline{\sigma_{\mathrm{ice}}}(t)+\overline{N U}(t)\right]$,

where

$\overline{\sigma_{\text {ice }}}(t)=\int_{\Omega_{0}} \sigma_{\text {ice }}(\Omega, t)\left[1-\mathcal{O}^{(0)}(\Omega)\right] \mathrm{d} \Omega$,

$\overline{N U}(t)=\int_{\Omega_{0}}[N(\Omega, t)-U(\Omega, t)] \mathcal{O}^{(0)}(\Omega) \mathrm{d} \Omega$,

and

$\overline{\mathcal{O}^{(0)}}=\int_{\Omega_{0}} \mathcal{O}^{(0)}(\Omega) \mathrm{d} \Omega$. 


\subsection{Time-varying ocean geometry}

To extend the theory for the possibility of time-varying ocean geometry, we now define the sea level height $h_{\mathrm{w}}$ mathematically as

$h_{\mathrm{w}}(\Omega, t)=h_{\mathrm{UF}}(t)+N(\Omega, t)-U(\Omega, t)-\zeta^{(0)}(\Omega)$,

which is the ocean water thickness at the point $\Omega$ and time $t$. We remind the reader that $\zeta^{(0)}(\Omega)$ stands for the SET at the initial time $t_{0}$. The function $h_{\mathrm{w}}$ is always positive over the ocean domain, and can be used to define the time-varying ocean function $\mathcal{O}(\Omega, t)$,

$\mathcal{O}(\Omega, t)=\left\{\begin{array}{l}1 \text { if } h_{\mathrm{w}}(\Omega, t)>0, \\ 0 \text { otherwise }\end{array}\right.$

Assuming again that ice and ocean water never overlap, the principle of equipotential surface gives the non-uniform contribution to the RSL height,

$s_{\mathrm{NU}}(\Omega, t)=[N(\Omega, t)-U(\Omega, t)] \mathcal{O}(\Omega, t)-\zeta^{(0)}(\Omega)\left[\mathcal{O}(\Omega, t)-\mathcal{O}^{(0)}(\Omega)\right]$.

According to eq. (17), the influence of the SET becomes important only for locations near the coast where the ocean function is time dependent. In the case that the ocean transgresses over time, the sea level height increases and, therefore, the ocean function changes from zero to one for a location in the flooded area. The second part of eq. (17) ensures that the RSL height is reckoned from the ocean bottom displaced by $U$ to the sea surface displaced by $h_{\mathrm{UF}}+N$. In the opposite case where the ocean regresses over time, the sea level height decreases and, therefore, the ocean function changes from one to zero for a location in the emerged area. The second part of eq. (17) again accounts for the fact that the RSL height is reckoned from the deformed ocean bottom to the deformed sea surface.

The principle of mass conservation, see eq. (10), now reads as

$$
\begin{aligned}
& \int_{\Omega_{0}} \sigma_{\text {ice }}(\Omega, t)[1-\mathcal{O}(\Omega, t)] \mathrm{d} \Omega+\varrho_{\mathrm{w}} h_{\mathrm{UF}}(t) \int_{\Omega_{0}} \mathcal{O}(\Omega, t) \mathrm{d} \Omega+\varrho_{\mathrm{w}} \int_{\Omega_{0}}[N(\Omega, t)-U(\Omega, t)] \mathcal{O}(\Omega, t) \mathrm{d} \Omega \\
& -\varrho_{\mathrm{w}} \int_{\Omega_{0}} \zeta^{(0)}(\Omega)\left[\mathcal{O}(\Omega, t)-\mathcal{O}^{(0)}(\Omega)\right] \mathrm{d} \Omega=0,
\end{aligned}
$$

which gives

$h_{\mathrm{UF}}(t)=-\frac{1}{\overline{\mathcal{O}}(t)}\left[\frac{1}{\varrho_{\mathrm{w}}} \overline{\sigma_{\mathrm{ice}}}(t)+\overline{N U}(t)-\overline{\zeta^{(0)}}(t)\right]$.

Here, $\overline{\sigma_{\text {ice }}}(t)$ and $\overline{N U}(t)$ are given by eq. (13), where $\mathcal{O}^{(0)}(\Omega)$ is replaced by $\mathcal{O}(\Omega, t)$, and

$\overline{\zeta^{(0)}}(t)=\int_{\Omega_{0}} \zeta^{(0)}(\Omega)\left[\mathcal{O}(\Omega, t)-\mathcal{O}^{(0)}(\Omega)\right] \mathrm{d} \Omega$.

Moreover,

$\overline{\mathcal{O}}(t)=\int_{\Omega_{0}} \mathcal{O}(\Omega, t) \mathrm{d} \Omega$.

\subsection{Floating ice}

So far, we have assumed that ice and ocean water never overlap (see Section 2.1). However, this cannot be guaranteed in all cases. For example, an ice sheet extending onto a continental shelf may still have a non-zero ice thickness over the ocean surface. The analysis of this case is discussed in the following.

Let us consider the situation where the ice column is heavier than the water column, that is, $\sigma_{\text {ice }}(\Omega, t) \geq \varrho_{\mathrm{w}} h_{\mathrm{w}}(\Omega, t)$. Archimedes' principle then implies that the water column is completely displaced by the ice column and water thickness is thus zero. In this case, the ice column is grounded and the point $\Omega$ is not subject to a water load at time $t$, and therefore it should not be included in the ocean area. In the other case where $\sigma_{\text {ice }}(\Omega, t)<\varrho_{\mathrm{w}} h_{\mathrm{w}}(\Omega, t)$, the ice floats and the water thickness is reduced by the mass of water displaced by the ice. To handle the two scenarios mathematically, we follow Lambeck et al. (2003) and introduce the water-thickness term, $h_{\mathrm{w}}^{*}(\Omega, t)$, by

$h_{\mathrm{w}}^{*}(\Omega, t)=h_{\mathrm{w}}(\Omega, t)-\frac{1}{\varrho_{\mathrm{w}}} \sigma^{\mathrm{ice}}(\Omega, t)$,

where $\sigma_{\mathrm{ice}} / \varrho_{\mathrm{w}}$ is the thickness of water displaced by the ice mass. The grounded and floating ice are then described by the respective inequalities $h_{\mathrm{w}}^{*}(\Omega, t) \leq 0$ and $h_{\mathrm{w}}^{*}(\Omega, t)>0$. The ocean function in eq. (16) generalized for the case of floating ice is

$\mathcal{O}(\Omega, t)=\left\{\begin{array}{l}1 \text { if } h_{\mathrm{w}}(\Omega, t)>0 \text { and } h_{\mathrm{w}}^{*}(\Omega, t)>0, \\ 0 \text { otherwise }\end{array}\right.$ 
Table 1. List of spherical cap ice-height models. See Fig. 1 for ice-height model L2.

\begin{tabular}{lccc}
\hline Model & $\vartheta_{0}$ & $\lambda_{0}$ & $h_{0}(\mathrm{~m})$ \\
\hline L0 & $0^{\circ}$ & $0^{\circ}$ & 1500 \\
L1 & $25^{\circ}$ & $75^{\circ}$ & 1500 \\
L2 & $25^{\circ}$ & $75^{\circ}$ & 500 \\
\hline
\end{tabular}

In view of the principle of equipotential surface, the non-uniform contribution to the RSL height is

$s_{\mathrm{NU}}(\Omega, t)=\left[N(\Omega, t)-U(\Omega, t)-\frac{1}{\varrho_{\mathrm{w}}} \sigma_{\mathrm{ice}}(\Omega, t)\right] \mathcal{O}(\Omega, t)-\zeta^{(0)}(\Omega)\left[\mathcal{O}(\Omega, t)-\mathcal{O}^{(0)}(\Omega)\right]$.

The principle of mass conservation, see eq. (10), now becomes

$$
\begin{aligned}
& \int_{\Omega_{0}} \sigma_{\text {ice }}(\Omega, t) \mathrm{d} \Omega+\varrho_{\mathrm{w}} h_{\mathrm{UF}}(t) \int_{\Omega_{0}} \mathcal{O}(\Omega, t) \mathrm{d} \Omega+\varrho_{\mathrm{w}} \int_{\Omega_{0}}[N(\Omega, t)-U(\Omega, t)] \mathcal{O}(\Omega, t) \mathrm{d} \Omega \\
& -\int_{\Omega_{0}} \sigma_{\text {ice }}(\Omega, t) \mathcal{O}(\Omega, t) \mathrm{d} \Omega-\varrho_{\mathrm{w}} \int_{\Omega_{0}} \zeta^{(0)}(\Omega)\left[\mathcal{O}(\Omega, t)-\mathcal{O}^{(0)}(\Omega)\right] \mathrm{d} \Omega=0,
\end{aligned}
$$

which gives $h_{\mathrm{UF}}$ in the same form as eq. (19). The reason is that the mass of the grounded ice calved at the coast of ocean in the case that ice and ocean do not overlap (see Section 2.2), that is, the mass

$\int_{\Omega_{0}} \sigma_{\text {ice }}(\Omega, t) \mathcal{O}(\Omega, t) \mathrm{d} \Omega$,

is equal to the mass of water displayed by floating ice (see Section 2.3), that is, the mass

$\int_{\Omega_{0}} \varrho_{\mathrm{w}} \frac{\sigma_{\text {ice }}(\Omega, t)}{\varrho_{\mathrm{w}}} \mathcal{O}(\Omega, t) \mathrm{d} \Omega$

All other terms in the principles of mass conservation (18) and (25) that are not proportional to $\sigma_{\text {ice }}$ are the same.

The ocean loads $h_{\mathrm{UF}}$ and $s_{\mathrm{NU}}$, and ocean function $\mathcal{O}$, given by eqs (19), (24) and (23), respectively, depend on the field variables $U$ and $N$ obtained by solving the viscoelastic field equations, which means that the forward modelling of GIA is represented by a free boundary-value problem. An ocean load is implemented as a boundary function over an ocean area with a free geometry, that is, an ocean load and the shape of ocean itself are being sought in addition to the deformation and gravitational-potential increment fields, by carrying out the forward modelling of GIA. We remind the reader that the theory presented in this section is the most general formulation we use in this paper and it includes the considerations presented in Sections 2.2 and 2.1 as special cases.

\section{BENCHMARK DESCRIPTION AND SETUP}

As introduced, this benchmark study focuses on testing and comparing various numerical implementations of the SLE in GIA numerical codes. This section describes the relatively simple synthetic examples that will serve this purpose. The setup consists of specifying the Earth model (Section 3.1), the ice load (Section 3.2), the surface topography (Section 3.3), and the consideration of fixed or moving coastlines (Section 3.4). From these, we choose five combinations defining the respective benchmark examples A to E (Section 3.5).

\subsection{Viscoelastic/density structure}

The reference Earth model for all benchmark examples is the spherically symmetric, five-layer, incompressible, self-gravitating viscoelastic earth model M3-L70-V01 (Spada et al. 2011, Table 3) used in the benchmark study of GIA numerical codes. The three mantle layers and the lithosphere of model M3-L70-V01 have a Maxwell viscoelastic and a purely elastic rheology, respectively, while the core is a uniform inviscid fluid.

\subsection{Ice load}

The surface density of ice-mass distribution, $\sigma_{\text {ice }}(\Omega, t)$, at point $\Omega$ and time $t$ is represented by the product of the height of ice at $(\Omega, t)$ and density of ice,

$\sigma_{\text {ice }}(\Omega, t)=\varrho_{\text {ice }} h_{\text {ice }}(\Omega, t)$.

The reference ice-height model for all benchmark examples is a spherical cap of height $h_{0}$ and radius $\alpha_{0}=10^{\circ}$ (Spada et al. 2011, Table 4, row 1) centred at co-latitude $\vartheta_{0}$ and longitude $\lambda_{0}$. Table 1 lists the three combinations of $\vartheta_{0}, \lambda_{0}$ and $h_{0}$ considered in this benchmark study. Model L0 was employed for the previously discussed benchmark for the various GIA numerical solvers (Spada et al. 2011). 


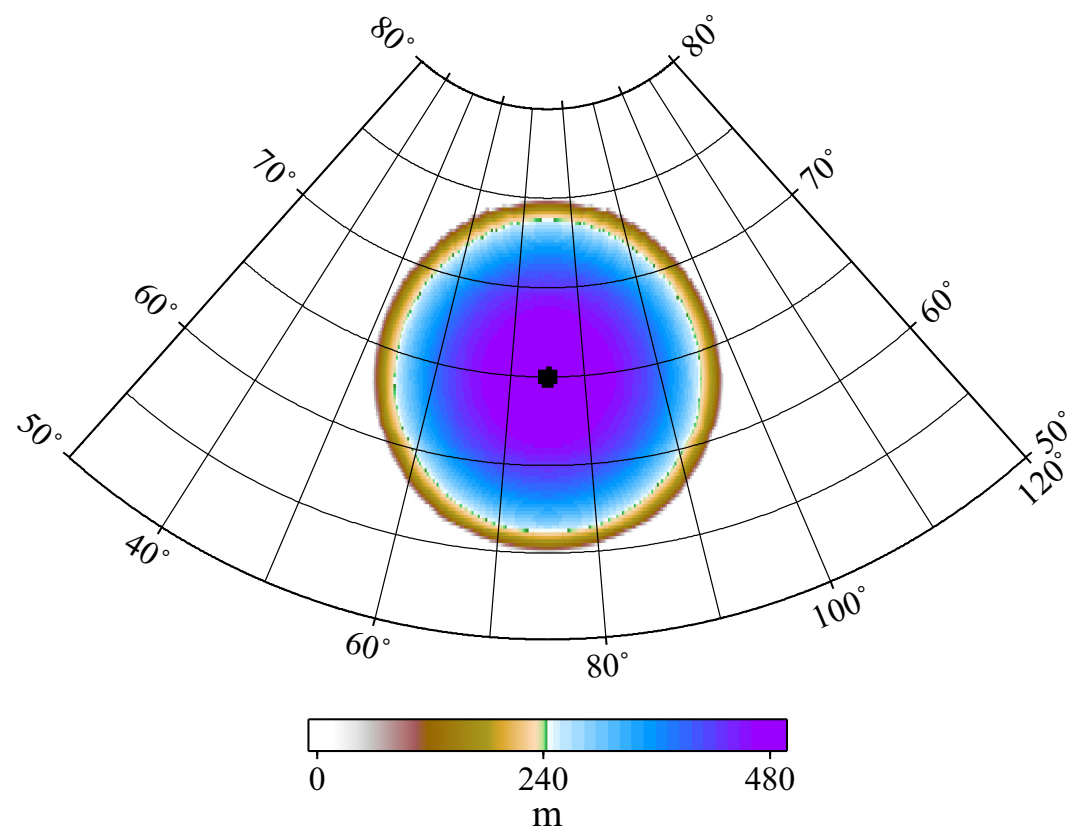

Figure 1. The ice-height model L2 (see Table 1).

Table 2. List of time scenarios of ice loading. The terminal time $t_{f}$ of numerical experiments is $t_{f}=t_{1}$ and $t_{2}$ for scenarios T0 and T1, respectively.

\begin{tabular}{ll}
\hline Scenario & Description \\
\hline T0 & Heaviside loading of the height of load, $h(t)$, and the radius of load, \\
& $\alpha(t)$, for $t \in\left(t_{0}, t_{1}\right], t_{1}=10 \mathrm{kyr}$, that is, $h(t)=h_{0}$ and $\alpha(t)=\alpha_{0}$. \\
T1 & A linear increase of $h(t)$ and $\alpha(t)$ for $t \in\left(t_{0}, t_{1}\right]$ such that \\
& $h\left(t_{0}\right)=0, h\left(t_{1}\right)=h_{0}, \alpha\left(t_{0}\right)=0$ and $\alpha\left(t_{1}\right)=\alpha_{0}$. For $t \in\left(t_{1}, t_{2}\right]$, \\
& $t_{2}=15 \mathrm{kyr}, h(t)=h_{0}$ and $\alpha(t)=\alpha_{0}$. \\
\hline
\end{tabular}

Table 3. List of the parameters of the ocean basin at the initial time $t=t_{0}$. See Fig. 2 for ocean-basin model B2.

\begin{tabular}{lcccc}
\hline Model & $\vartheta_{b}$ & $\lambda_{b}$ & $b_{\max }(\mathrm{m})$ & 0 \\
\hline B0 & $0^{\circ}$ & $0^{\circ}$ & 0 & $b_{0}(\mathrm{~m})$ \\
B1 & $100^{\circ}$ & $320^{\circ}$ & 760 & 1200 \\
B2 & $35^{\circ}$ & $25^{\circ}$ & 3800 & 6000 \\
\hline
\end{tabular}

Table 4. List of the benchmark cases carried out in this work. The ocean basin B2* is the same as B2, but the SET $\zeta^{(0)}$ in benchmark E is prescribed at the terminal time $t_{\mathrm{f}}$.

\begin{tabular}{lccccc}
\hline Specification & A & B & Benchmark cases & D & E \\
\hline SLE & 0 & 1 & 1 & 2 & L2 \\
Load spatial & L0 & L1 & L2 & T1 & T1 \\
Load temporal & T0 & T0 & T1 & B2 & B2* \\
Ocean basin & B0 & B1 & B2 & \\
\hline
\end{tabular}

The ice-height model L2 with a relatively low elevation is designed such that when it reaches the coast of the ocean basin, the condition of floating ice, that is, $h_{\mathrm{w}}^{*}(\Omega, t)>0$, is satisfied. This allows for the demonstration of the effect of floating ice when solving the SLE, which is done by the comparison with the results of the benchmark example for the relatively thick ice model L1. Fig. 1 illustrates the ice-height model L2, where ice reaches a height of $500 \mathrm{~m}$ at the point $\left(25^{\circ}, 75^{\circ}\right)$.

In addition, the spherical ice cap is allowed to vary over time according two time scenarios, which are listed in Table 2. A Heaviside loading scenario T0 is used in the first benchmark example, where the SLE is not included in the GIA modelling so as to find an initial agreement between all individual solutions. Time scenario T1 is designed for testing the GIA solutions with the SLE, either for a fixed or time varying ocean geometry, and the case where inland ice is advancing over time and reaches the coast of the ocean basin. 


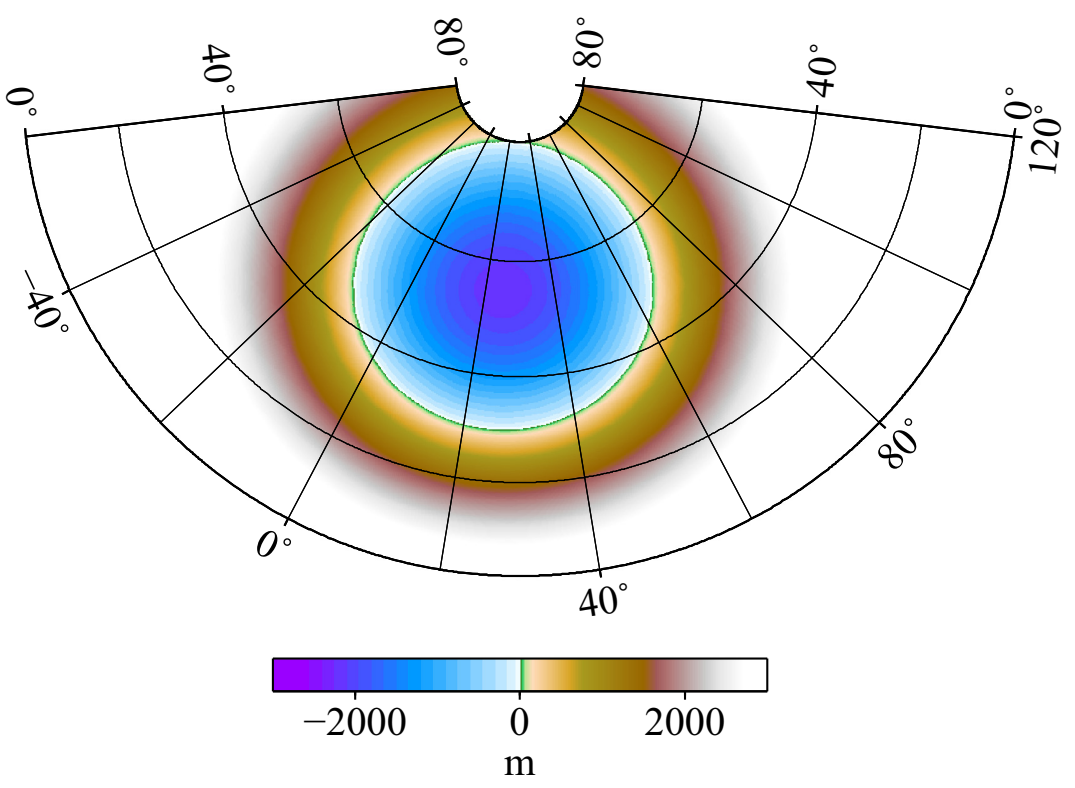

Figure 2. The solid Earth topography with the ocean-basin model B2 (in blue) (see Table 3).

\subsection{Initial SET}

The SET at the initial time $t_{0}$, denoted by $\zeta^{(0)}(\Omega)$ in Section 2 , is defined by a circular exponential basin centred at co-latitude $\vartheta_{b}$ and longitude $\lambda_{b}$, that is, by the function

$\zeta^{(0)}(\Omega)=b_{\max }-b_{0} \exp \left\{-\psi^{2} / 2 \sigma_{b}^{2}\right\}$,

where

$\cos \psi=\cos \vartheta \cos \vartheta_{b}+\sin \vartheta \sin \vartheta_{b} \cos \left(\lambda-\lambda_{b}\right)$

and $\sigma_{b}=26^{\circ}$ for all benchmark examples. The other parameters of the ocean basin are chosen according to the test examples and are listed in Table 3. The function $\zeta^{(0)}(\Omega)$ has the minimum of $b_{\max }-b_{0}$ at the centre of the ocean basin $\left(\psi=0^{\circ}\right)$ and the maximum of $b_{\max }$ at the antipole $\left(\psi=180^{\circ}\right)$. The radius of the ocean basin is $\psi_{0}=\sigma_{b} \sqrt{\ln \left(b_{0} / b_{\max }\right)^{2}}$, which is equal to $\psi_{0}=24.85^{\circ}$ for both ocean basins $\mathrm{B} 1$ and B2. Model B0, that is, the model without ocean loading, was employed in the previous benchmark study (Spada et al. 2011). The ocean basin B1 is placed at a far distance from the ice load, allowing for the demonstration of the effect of inland ice on solving the SLE, considering either a fixed or time-varying ocean geometry. The ocean basin B2 is relatively deep and is placed near the ice load, which allows for the demonstration of the effect of floating ice on solving the SLE. Fig. 2 illustrates $\zeta^{(0)}(\Omega)$ for the ocean-basin model B2. The ocean reaches a depth of $2200 \mathrm{~m}$ at point $\left(35^{\circ}, 25^{\circ}\right)$.

\subsection{Sea level equation}

Concerning the complexity of the SLE, the benchmark examples can be classified into two test suites, referred to as SLE $=1$ and SLE=2, described in Sections 2.1 and 2.3, respectively. The two cases are characterized by the fixed and time-varying ocean geometries, respectively. The case SLE $=0$ corresponds to the benchmark study by Spada et al. (2011), where the SLE is not included when carrying out the forward modelling of GIA.

\subsection{Benchmarks}

There are five benchmark examples presented in this work, denoted as A-E in increasing complexity. They differ in the spatial distribution and time evolution of the ice load: whether an inland or marine-terminated ice sheet is prescribed, the depth of the ocean basin, and a fixed or time-varying ocean geometry (see Table 4). Benchmark A represents the simplest setup, in which case the SLE is not solved. This model has been previously employed to evaluate various GIA numerical solvers (Spada et al. 2011) and is therefore a convenient starting point for the numerical comparisons in our exercise. In benchmark B, the ice load is placed in the interior of a continent such that it never reaches the ocean basin (see Fig. 4), and the SLE is solved with a fixed ocean geometry. In benchmark C, the ice load is placed near the ocean basin such that when it is advancing over time, it reaches the coast of the ocean basin. The SLE is still solved with a fixed ocean geometry, that is, the ice load is calved when reaches the coast (see Fig. 7). Benchmark D again considers the marine-terminated ice (see Fig. 12), but the SLE is now solved with a time-varying ocean geometry. Finally, the setup of benchmark E is the same as that of benchmark D, but, instead of the initial 
Table 5. List of numerical methods with the independently developed numerical codes for GIA modelling with the SLE that are evaluated in this paper. The abbreviations for numerical parametrizations: TD, time-domain; LD, Laplace-domain; SH, spherical harmonics; FD, finite differences; FE, finite elements.

\begin{tabular}{lllll}
\hline Abb. & Code & Author or User & Parametriz. & References \\
\hline ZM & VEGA & Z. Martinec & TD-FE-SH & Martinec (2000) \\
VK & VILMA & V. Klemann & TD-FE-SH & Klemann et al. $(2008)$ \\
MS & SELEN & D. Melini, G. Spada & LD-FD-SH & Spada \& Stocchi (2007) \\
WW & TUDSLE & W. van der Wal & LD-FD-SH & Schotman (2007) \\
YS & MUSSEL & Y. Sun, R. E. M. Riva & LD-FD-SH & Riva et al. $($ 2018) \\
KS & PGCcalc & K. Simon, T. James & LD-FD-SH & James \& Ivins (1998) \\
VB & TSec02 & V. R. Barletta & LD-FD-SH & Barletta \& Spada (2012) \\
WWa & AFCAL & W. van der Wal & TD-FE-FE/SH & Wu \& van der Wal (2003) \\
GA & A13 & G. A & LD-FD-SH & A et al. $(2013)$ \\
SK & giapy & S. Kachuck & TD-FD-SH & Kachuck (2017) \\
\hline
\end{tabular}

SET, its geometry at the terminal time is prescribed as a input parameter. The initial SET is now searched for, such that its geometry at the terminal time is adjusted. The motivation to carry out benchmark E is that the initial SET is less well known than the current SET, which can be measured.

\section{SOLUTION METHODS AND NUMERICAL CODES}

The mathematical and numerical methods employed in this study have been previously validated and published in a suite of independent publications. For the sake of completeness, we list these methods here, and refer the interested reader to the relevant publications for additional details. For ease of presentation, the numerical codes we evaluate in this work and the corresponding abbreviations are summarized in Table 5 (note that in the following we will use the abbreviations listed in Table 5). Concerning time parametrization, the codes developed by ZM, VK, SK and WWa are time-domain solvers. All other codes use the viscoelastic surface load Love numbers, which are computed in the Laplace domain. The methods of spatial parametrization fall, broadly, into three distinct categories: finite elements (WWa), spherical harmonic-finite elements (ZM, VK) and spherical harmonic-finite differences (WW, YS, MS, KS, VB, GA, SK).

In the following sections, common and particular aspects of each method are highlighted, and the reader is referred to previous papers in which these schemes are described in detail.

\subsection{VEGA and VILMA}

Benchmark computations by ZM and VK have been done by the VEGA and VILMA numerical codes, respectively. The VEGA code is based on the spectral-finite elements formulation of the viscoelastic field equations describing the deformations of a self-gravitating spherical body under a surface load developed by Martinec (2000). The SLE was implemented in VEGA during this benchmark study. The VILMA code originates from VEGA, but was further developed by implementing the SLE (Hagedoorn et al. 2007), rotational deformation (Martinec \& Hagedoorn 2005) and the coupling with a dynamic ice-sheet model (Konrad et al. 2014, 2016). Furthermore, the code was modularised in FORTRAN 90, and the restart functionality was implemented for running on high-performance computing facilities. Due to the separate development of VILMA from the original version of VEGA, we consider VILMA to be an independent numerical tool, and, therefore, a valuable contributor to this benchmark.

VEGA and VILMA use the spherical harmonic (SH) parametrization of the viscoelastic field equations. For VEGA, the SH parametrization is from degree zero up to degree and order 256 (except for benchmark A, where the solution starts at degree 2). The finite element (FE) parametrization of field variables in the radial direction contains 165 piecewise-linear FEs with radial steps of 5,10 and $40 \mathrm{~km}$ in the lithosphere, upper and lower mantle, respectively. For VILMA, the SH parametrization extends up to degree and order 256 for benchmarks A and E; otherwise, up to degree and order 128. The lithosphere and upper mantle, the transition zone, and the lower mantle are discretised with the radial step of $2.5,10$ and $40 \mathrm{~km}$, respectively. In total, there are 248 FEs. In order to mimic the fixed coastlines in benchmarks B and $\mathrm{C}$, the bathymetry is replaced by a step function with $\pm 500 \mathrm{~m}$ water depth or land height. This is a necessary arrangement as VILMA does not have a possibility to avoid a moving-coastline case. For both codes, the surface load distribution and SET are represented on the spatial grid $G$ of 1024 Gauss-Legendre latitudinal nodes and 2048 equiangular longitudinal nodes, $G=1024 \times 2048$. The SLE is solved pointwise on $G$ by an explicit time-differencing scheme with a time step of $20 \mathrm{yr}$, which is the same as for the viscoelastic field equations.

\subsection{SELEN}

Benchmark computations by DM and GS have been obtained using two different numerical codes, TABOO and SELEN, both of which are based on the classical VNM method. The results of benchmark A have been obtained with the TABOO post-glacial rebound code (Spada et al. 2004). TABOO computes the viscoelastic Love numbers (VLNs) for a spherically symmetric, incompressible, layered earth model; it has been successfully tested with earth models containing up to nine viscoelastic layers, and for SH degrees of the order of a few hundred. 
Beyond those limits, numerical instabilities may arise in relation to the computation of the relaxation modes. TABOO can use the computed VLNs to obtain the time-dependent response to a complex ice load, but it does not solve the full SLE. For the purposes of benchmark A, a modified version of the original TABOO code was used, originally developed for the GIA benchmark by Spada et al. (2011) ${ }^{2}$. A suite of load geometries and time histories are handled by TABOO analytically, and therefore no spatial or temporal discretization is invoked.

The results of the other four benchmarks, B-E, have been obtained with a new version of SELEN (version 4), which is a general code for solving the SLE (Spada \& Stocchi 2007). SELEN incorporates TABOO for the VLNs computation, inheriting the limitations described above, and obtains a gravitationally self-consistent response to a prescribed ice load by iteratively solving the SLE in the SH domain. A new version of SELEN includes, among other features, a rotational feedback according to Milne \& Mitrovica (1998) and migrating coastlines (according to the formulation of Mitrovica \& Milne (2003)), and properly handles the transition between floating and grounded ice sheets. SELEN internally represents time-dependent fields as piecewise constant functions, with a time-step of $0.5 \mathrm{kyr}$. The spatial domain is discretized in SELEN with an equal-area, icosahedron-based grid of disc-shaped pixels (Tegmark 1996), which represents a natural quadrature set for the SH representations of fields. The spatial grid employed in benchmarks B-E consists of 75692 pixels, with each pixel corresponding to a disc of radius $46.3 \mathrm{~km}$. This grid ensures accurate numerical integration of harmonic functions up to a degree of about 470 (Tegmark 1996). All the SELEN computations have been performed by five iterations of the SLE.

\subsection{TUDSLE}

The code developed by Schotman (2007) in Fortran 90 is based on the pseudo-spectral SLE (Mitrovica \& Peltier 1991), including timedependent continental margins and near-field water dumping as described by Milne et al. (1999). It is combined with a code for rotational feedback implemented by van Dael (2006). Adjustments have been made for different ice loading histories and for better convergence when solving for a self-consistent sea level and for pre-glacial topography. An earlier version of the code produced results that matched well with the output from a FE model (Wu \& van der Wal 2003). The code was a part of the benchmark of the SLE by Barletta et al. (2013). Recently, the code has been extended to include sediment loading as surface forcing (van der Wal \& IJpelaar 2017). The code requires as input a file with surface load Love numbers, which were produced with a normal mode code benchmarked in Spada et al. (2011). For the ice load and topography files, an equiangular grid was used with $0.03125^{\circ}$ and $0.25^{\circ}$ grid spacing for benchmarks A and B, respectively. Time step is equal to 1 kyr. For the tests which include self-consistent sea levels the iteration is stopped when the average relative difference is below a threshold. This results in a maximum of 6 iterations per time step.

\subsection{PGCcalc}

Benchmark computations by KS were carried out by the numerical code PGCcalc (Post Glacial Changes calculator) that solves the viscoelastic field equations in the pseudo-spectral domain using the methods described in James (1991) and James \& Ivins (1998). Decay times of incompressible spherically symmetric layered earth models are found by the VNM method. The SLE is solved self-consistently following the methods of Mitrovica \& Milne (2003) and Kendall et al. (2005). Tests of the implementation of marine-based ice sheets show agreement with previously published results for Antarctica (Simon et al. 2010) and North America (Simon et al. 2016). Although static shorelines are used in benchmarks A and B performed by the code PGCcalc in this study, the code has also been adapted to allow for fully migrating coastlines over time, and the code was part of a previous benchmark study on the SLE (Barletta et al. 2013). The ice load is represented on a $0.125^{\circ}$ equiangular grid in both benchmarks A and B. Calculations in the spectral domain are performed to SH degree and order 256.

\section{5 giapy}

The giapy package ${ }^{3}$ is an open-source python package for computing glacial isostatic adjustment on a spherically symmetric, viscoelastic earth model. The SLE solver (giapy . sle) convolves viscoelastic response curves to SH loads, transforming back and forth from the Legendre and spatial domains using the pyspharm python binding of SPHEREPACK (Whitaker 2016). The package includes a time-domain, FD solver for the spherical-harmonic viscoelastic Love numbers. As this solver is in the process of being benchmarked, time-domain results from TABOO were used in this paper for the earth response.

For each $20 \mathrm{yr}$ time step, the ice load increment and ocean load redistribution, consistent with shifting coastlines in the manner of Mitrovica \& Milne (2003), is computed using the scipy root function (Jones et al. 2001) to find the $h_{\mathrm{UF}}$ that minimizes the excess mass. The instantaneous elastic response is then computed, iterating the elastic response and ocean geometry until an additional iteration results in less than 1 per cent average surface change. Changes in topography, sea-surface variation, and ocean function are always kept in the spatial domain, with loads transformed to and responses transformed from the Legendre domain. The spatial domain has been discretized into an equiangular grid with 721 latitudes and 512 longitudes.

\footnotetext{
${ }^{2}$ The TABOO code is freely available from https://github.com/danielemelini/TABOO.

${ }^{3}$ https://github.com/skachuck/giapy
} 


\subsection{MUSSEL}

The MUSSEL solver, used by YS, is an updated version of the code developed at the Delft University of Technology (Schotman \& Vermeersen 2005; Riva et al. 2010). It is based on the pseudospectral SLE (Mitrovica \& Peltier 1991) and it includes migrating coastlines (Mitrovica \& Milne 2003). This solver has been harmonized and largely re-written within the context of the MUSSEL project ${ }^{4}$, while some features were added specifically for this study, such as the possibility to prescribe the SET at the present time (benchmark E). The VLNs necessary to determine the solid earth response are computed separately and provided as input. Computations are performed in the spectral domain up to SH degree 256 and in the spatial domain on a Gaussian grid of 1024 by 2048 nodes. Time steps are always equal to 100 yr for Heaviside loads applied at the beginning of the time evolution.

\subsection{TSec02}

The code TSec02 developed in Fortran 90 (Barletta \& Spada 2012) is based on the pseudo-spectral SLE (Mitrovica \& Peltier 1991). The code calls the TABOO solver to compute the viscoelastic Love numbers for a spherically symmetric, incompressible, layered earth model. The transformation between the spatial and SH domains is performed by the fast Fourier transform. The code was a part of the benchmark of the SLE (Barletta et al. 2013).

\section{$4.8 \mathrm{~A} 13$}

GA utilizes a semi-analytic model, referred to as A13 hereafter, that solves for the viscoelastic response of an incompressible earth model to surface loading (A et al. 2013). A13 assumes a spherically symmetric earth model and accounts for degree-one motion, self-gravitation, dynamic ocean loading, and polar wander feedback. The convolution between the Love numbers and surface loads are carried out in the Laplace domain, and changes in geoid, radial and horizontal surface deformation are all calculated in the SH domain. This model has been benchmarked with an FE model which is designed to calculate the viscoelastic response of a laterally heterogeneous Earth (A et al. 2013). A13 calculates the dynamic ocean load in an iterative manner. At each epoch, the model first solves for the Earth's response by assuming a static ocean load along with zero perturbations in the geoid and solid Earth's topography. The resulting changes in the geoid and surface topography are then used to determine a new ocean load. This process is repeated until the results converge, which generally takes fewer than three iterations.

\subsection{AFCAL}

The code AFCAL (Wu \& van der Wal 2003; van der Wal et al. 2010) is an axisymmetric FE model coupled with the SH method of solving the Laplace equation. It is the only method in this study that uses FEs in the tangential direction. The viscoelastic deformation of an earth model is computed by the FE program ABQAQUSTM, where the buoyancy forces are applied as the Winkler foundations at the internal density interfaces. These deformations are then used to solve the Laplace equation in the SH domain for the determination of gravitational potential increment. The viscoelastic displacement is expanded up to degree and order 120. We noted that increasing the degree to 150 does not have a significant effect on results. The change in gravitational potential is applied as boundary-value data at the layers in the Earth model after which the computation proceeds iteratively (Wu 2004). Four iterations are used to compute the final results. The self-consistent sea levels can, in principal, be included in the code, but the code considers an axially symmetric ice cap and oceans, the SLE is not applied here. The FE model uses the 4-node bilinear axisymmetric quadrilateral elements. The internal layer boundaries are placed at depths of $0,35,70,140$, $220,310,420,545,670,890,1110,1330,1550,1770,1990,2210,2430,2650,2891 \mathrm{~km}$, such that each layer in the Earth model contains at least two FE layers. The horizontal size of the elements is $0.25^{\circ}$. The Poisson ratio is set to 0.495 to avoid volumetric locking. Using the Poisson ratio of 0.4999 with hybrid elements to avoid volumetric locking does not give a significant difference in the results. The function of the ice thickness is evaluated at the centre of the nodes. The average of two nodes is used to calculate a distributed load that is applied at the centre of an element.

\section{RESULTS}

The benchmark study focused on testing and comparing various numerical implementations of the SLE in GIA numerical codes. This section describes the results of numerical runs for the benchmark examples specified in Section 3.

We should first emphasize that any independent numerical tool is a valuable contribution to the benchmark study. Hence, no restrictions have been placed on the solution methods listed in Table 5. However, not all tested codes produce the necessary output to fill all the entries of Table 6 . Hence, the suite of tests will be continued and the results published in a specifically designed web page ${ }^{5}$.

\footnotetext{
${ }^{4}$ https://www.nwo.nl/en/research-and-results/research-projects/i/68/10368.html

${ }^{5} \mathrm{https}: / /$ geofjv.troja.mff.cuni.cz/GIABenchmark/
} 
Table 6. The contributions to the benchmark examples so far.

\begin{tabular}{|c|c|c|c|c|c|c|c|c|c|c|}
\hline \multirow[b]{2}{*}{ Example } & \multicolumn{10}{|c|}{ Participant } \\
\hline & $\mathrm{ZM}$ & VK & YS & MS & SK & WW & VB & $\mathrm{KS}$ & GA & WWa \\
\hline A & $\mathrm{x}$ & $\mathrm{x}$ & $\mathrm{x}$ & $\mathrm{x}$ & $\mathrm{x}$ & $\mathrm{x}$ & $\mathrm{x}$ & $\mathrm{x}$ & $\mathrm{x}$ & $\mathrm{x}$ \\
\hline B & $\mathrm{x}$ & $\mathrm{x}$ & $\mathrm{x}$ & $\mathrm{x}$ & $\mathrm{x}$ & $\mathrm{x}$ & $\mathrm{x}$ & $\mathrm{x}$ & $\mathrm{x}$ & \\
\hline $\mathrm{C}$ & $\mathrm{x}$ & $\mathrm{x}$ & $\mathrm{x}$ & $\mathrm{x}$ & $\mathrm{x}$ & & & & & \\
\hline $\mathrm{D}$ & $\mathrm{x}$ & $\mathrm{x}$ & $\mathrm{x}$ & $\mathrm{x}$ & $\mathrm{x}$ & & & & & \\
\hline $\mathrm{E}$ & $\mathrm{x}$ & $\mathrm{x}$ & $\mathrm{x}$ & $\mathrm{x}$ & $\mathrm{x}$ & & & & & \\
\hline
\end{tabular}

Four scalar field variables, that is, three components of displacement and the gravitational potential increment, result from the forward modelling of GIA. From these, only the surface vertical displacement $U$ and geoid displacement $N$ enter the sea-surface variation $S$ and the uniform contribution $h_{\mathrm{UF}}$ to the RSL height. We will use $U, N, S$ and $h_{\mathrm{UF}}$ to compare the individual numerical solutions and assess the differences between them. The comparisons will be done along the latitudinal cross-sections passing through the centre of the ice cap and the centre of the ocean basin. For a better understanding of the results, we will also show the surface distribution of $U$ at the terminal time of the computations and the time evolution of the thickness of the uniform water layer, $h_{\mathrm{UF}}(t)$, given by eqs (12) and (19) for a fixed and time-varying ocean geometry, respectively.

The performance of 10 numerical codes are compared in a series of five benchmark exercises with an increasing degree of complexity as specified in Table 4. As mentioned in Section 1, because of the complexity of the SLE, no analytical solution to it exists. Hence, the accuracy of the considered numerical implementations is assessed by the differences of the individual solutions with respect to a reference solution. For that, we choose the solution provided by VEGA. In the Appendix, the convergence behaviour of the VEGA solution for increasing resolution of SH parametrization of the GIA field variables and increasing resolution of the spatial grid for solving the SLE is demonstrated. The reference solution for the comparisons is the VEGA solution calculated for the input parameters given in Section 4.1. We denote this by VEGA $^{\text {ref. }}$.

The differences in a quantity $q(U$ or $N)$ with respect to a reference quantity $q^{\text {ref }}$ will be quantified by two difference metrics,

$\epsilon_{q}^{\mathrm{RMS}}=\sqrt{\frac{1}{n} \sum_{i=1}^{n}\left(q_{i}-q_{i}^{\mathrm{ref}}\right)^{2}}, \quad \epsilon_{q}^{\mathrm{MAX}}=\max _{i}\left|q_{i}-q_{i}^{\mathrm{ref}}\right|$,

where $q_{i}$ and $q_{i}^{\text {ref }}$ are the results of an individual solution and a reference solution, respectively, at a given spatial location $i(i=1, \ldots, n)$ within a cross-sectional interval and fixed time. $\epsilon_{q}^{\mathrm{RMS}}$ and $\epsilon_{q}^{\mathrm{MAX}}$ are the root-mean square difference and maximum difference for quantity $q$, respectively. For each benchmark example, we provide graphical comparisons of $U$ and $N$ (and also $S$ for the cross-sections passing through the centre of the ocean basin) supported by plotted calculations of the differences.

\subsection{Benchmark A: no SLE}

Benchmark A does not include the SLE in the boundary condition for a surface load in the GIA model. In fact, this case coincides with the example performed in the benchmark study by Spada et al. (2011,Test 1/2). However, it played an important role in the current study since it helped the participants to correctly define the Earth's input parameters, choose the representative outputs of the calculations and reach a good agreement between the results of all participants, especially those who did not take part in the benchmark by Spada et al. (2011). More importantly, benchmark A establishes the level of inconsistencies between individual numerical results, which can be later used for testing whether the inconsistencies are getting worse with increasing complexity of the SLE in subsequent tests.

The left-hand panels of Fig. 3 show the latitudinal cross-section of surface vertical displacement $U$ and geoid displacement $N$ through the centre of the ice cap at $t=10 \mathrm{kyr}$. Note that the ice load components of degree 0 and 1 have not been considered in this first example. Inspecting the left-hand panels of Fig. 3 we can see that the minimum values of $U$ and $N$ are situated at the centre of the ice cap, while the maximum of $U$ is at a distance of $14.1^{\circ}$ from the centre of the ice cap, where the forebulge has formed. This is a known response of viscoelastic material to Heaviside loading (e.g. Spada et al. 2011). All solutions match very well. This agreement is particularly valuable since this is the only benchmark example considered by all participants, who provided computations based on a wide range of independently developed codes.

To get a more quantitative view on comparison, the right-hand panels of Fig. 3 show the differences between the individual solutions and the reference solution VEGA $^{\text {ref }}$ along the co-latitudinal interval $\left(0^{\circ}, 20^{\circ}\right)$. We can see that the differences have a medium-wavelength feature with large spikes at the edge of the ice cap at a co-latitude of $\vartheta=10^{\circ}$. The spikes are an artefact of the spectral representation of the ice cap. That is, it has an infinite slope at its foot and, consequently, a truncated SH series representing the ice cap oscillates in proximity of this point and converges extremely slowly with increasing cut-off degree. When the truncated SH series of the load is processed by a numerical code, the viscoelastic response of an earth model oscillates in the vicinity of the foot of the ice cap. Though the oscillations are dampened, they are not completely filtered out by the earth model. In principle, this effect is the same for each approach applied here. However, the various numerical precisions of the individual numerical codes and the conversion between the spatial grids are mostly responsible for the differences shown in the right-hand panels of Fig. 3. The metrics of the differences are graphically shown in the first columns of Fig. 20. Except for SK, 
Benchmark A ( $\mathrm{t}=10 \mathrm{kyr})$
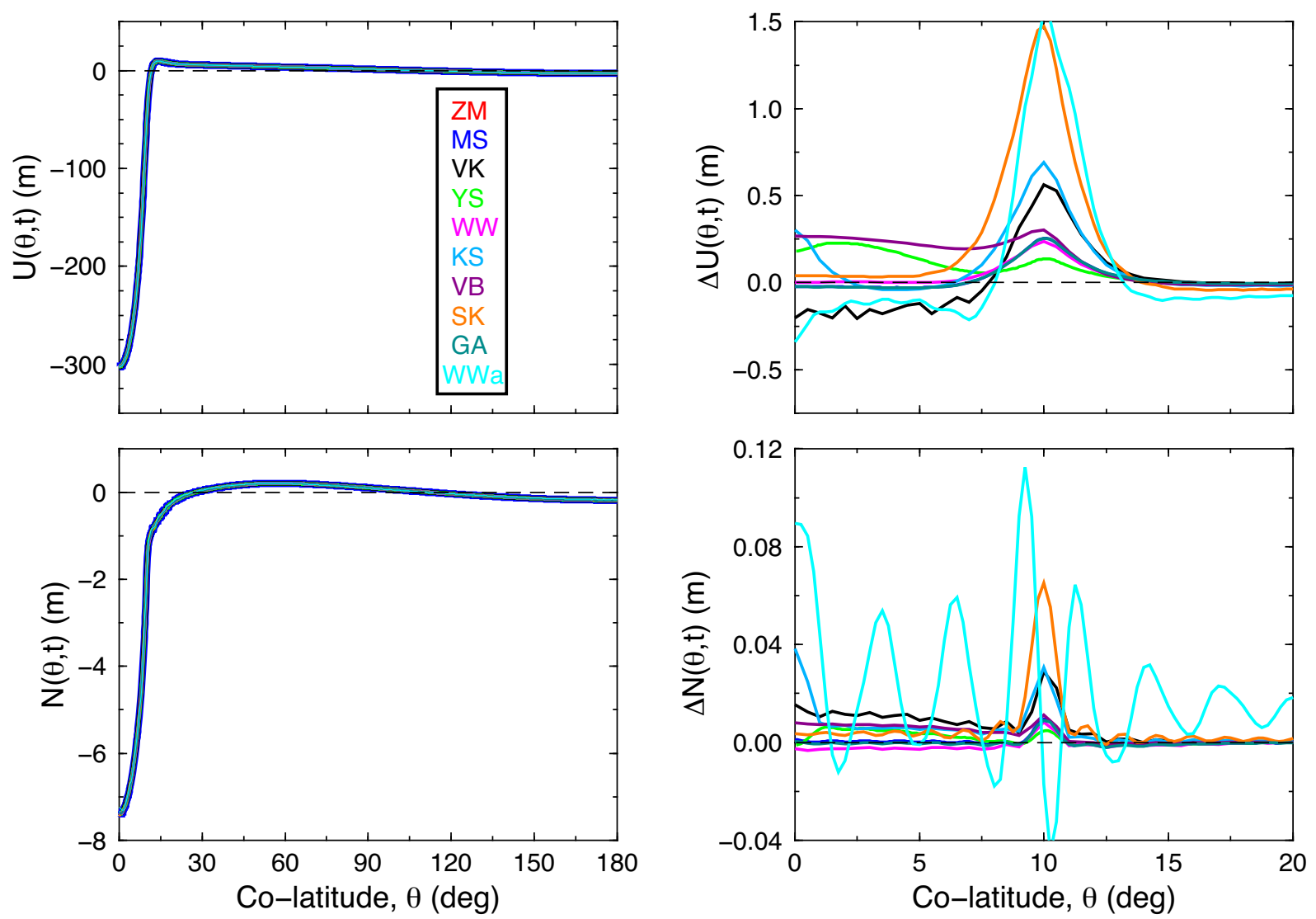

Figure 3. Left-hand panels: the latitudinal cross-section of surface vertical displacement $U$ and geoid displacement $N$ through the centre of the ice load located at $\left(0^{\circ}, 0^{\circ}\right)$ and time $t=10 \mathrm{kyr}$ for benchmark A provided by the participants of the benchmark study. The abbreviations are shown in Table 5 . Right-hand panels: the differences between the individual solutions and $\operatorname{VEGA}^{\text {ref }}$ (Section 4.1) along the co-latitudinal interval $\left(0^{\circ}, 20^{\circ}\right)$. The root-mean square and maximum differences of the individual solutions are shown in Fig. 20.
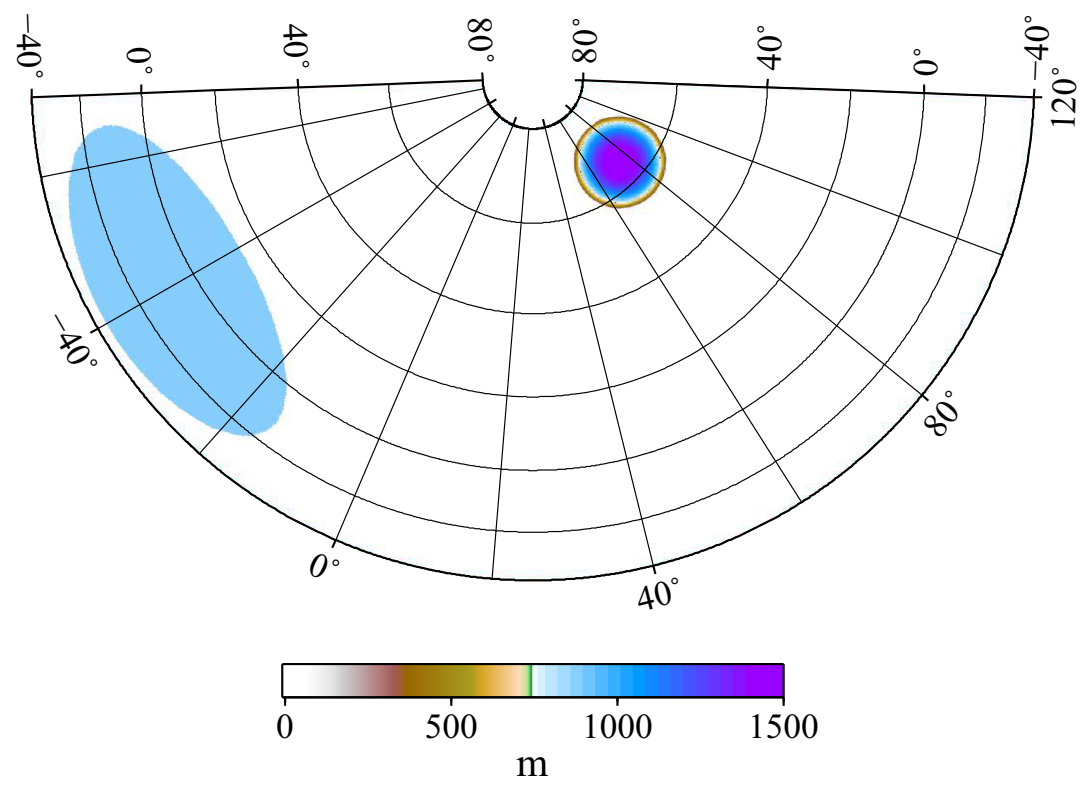

Figure 4. Height of the grounded ice at $t=10 \mathrm{kyr}$ in benchmark B computed by VEGA ${ }^{\text {ref }}$. The grounded ice does not reach the ocean basin during the loading period. The light blue ellipse shows the circular ocean function, which is fixed throughout time. 


\section{Benchmark $B\left(\lambda=75^{\circ}, t=10 \mathrm{kyr}\right)$}
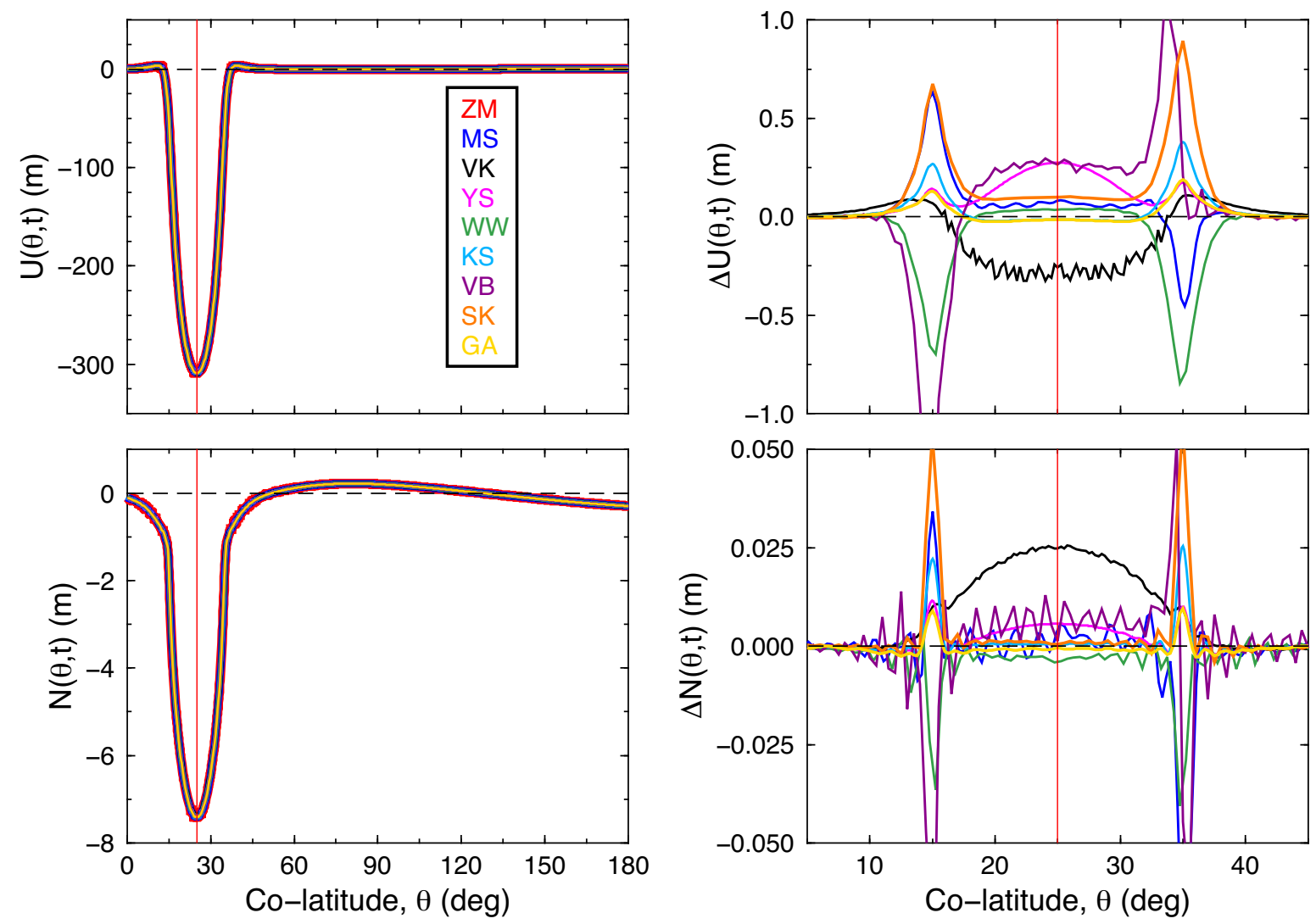

Figure 5. The same as Fig. 3, but for benchmark B. The centre of the ice load is located at $\left(25^{\circ}, 75^{\circ}\right)$, which is indicated by the red vertical line.

all other solutions differ maximally by 0.7 and $0.03 \mathrm{~m}$ in vertical and geoid displacement, respectively (which is relatively 1.5 per cent), with the root-mean square differences up to 0.2 and $0.01 \mathrm{~m}$, respectively.

The AFCAL model underestimates the maximum deflection at $10 \mathrm{kyr}$ compared to the other models (Fig. 3, top left and right). The difference at the point of maximum displacement is $0.3 \mathrm{~m}$ which amounts to 0.1 percent. The largest difference of $1.7 \mathrm{~m}$ is at the edge of the load. The difference in the geoid displacement shows oscillatory behaviour with a wavelength of about $300 \mathrm{~km}\left(\right.$ or $3^{\circ}$ ) and a maximum amplitude of $0.11 \mathrm{~m}$. The discretization of acting forces and linear deformation of FE elements is a common source of errors of FE-based codes. A further reduction of the difference with respect to the other methods could be achieved by reducing the FE size, especially near the edge of the load, and using quadratic elements. However, such a refinement greatly increases computation time of the FE method. The resolution achieved here is comparable with the resolution that can be achieved for a global GIA model.

\subsection{Benchmark B: fixed coastlines, inland ice sheet}

In benchmark B, the ice with a Heaviside loading history is placed in the interior of the continent, such that it never reaches the ocean basin during the glaciation period, and the SLE is solved with a fixed ocean geometry. This is illustrated in Fig. 4, where the height of grounded ice at the terminal time $t=10 \mathrm{kyr}$ is shown. The ocean function drawn by the light blue ellipse is fixed throughout time.

The left panels of Fig. 5 show the latitudinal cross-section of surface vertical displacement $U$ and geoid displacement $N$ through the centre of the ice cap at $t=10 \mathrm{kyr}$. In contrast to benchmark A, the ice load components are taken from SH degree 0 up to degree 256 in the computation. Comparing Fig. 5 with Fig. 3, we can see that $U$ and $N$ behave similarly with respect to the centre of the ice cap as those in benchmark A. The displacement $U$ again reaches its maximum at the distance of about $14.1^{\circ}$ from the centre of the ice cap, where the forebulge has formed. The left-hand panels of Fig. 5 show the excellent agreement between the individual solutions.

The differences between the individual solutions and $\mathrm{VEGA}^{\text {ref }}$ along the co-latitudinal interval $\left(5^{\circ}, 45^{\circ}\right)$ are shown in the right-hand panels of Fig. 5. The large spikes at the edges of ice cap are due to the same reason as for Fig. 3. The metrics of the differences are graphically shown in the second columns of Fig. 20. Except for VB, all other differences are comparable to those of benchmark A. However, we can see that the differences for the MS, VB and WW solutions are now larger compared to those for benchmark A, while the other solutions are smaller (SK) or slightly smaller.

Fig. 6 shows the latitudinal cross-section of $U, N$ and $S$ through the centre of the ocean basin at $t=10$ kyr. We can see that the surface 
Benchmark $B\left(\lambda=320^{\circ}, t=10 \mathrm{kyr}\right)$
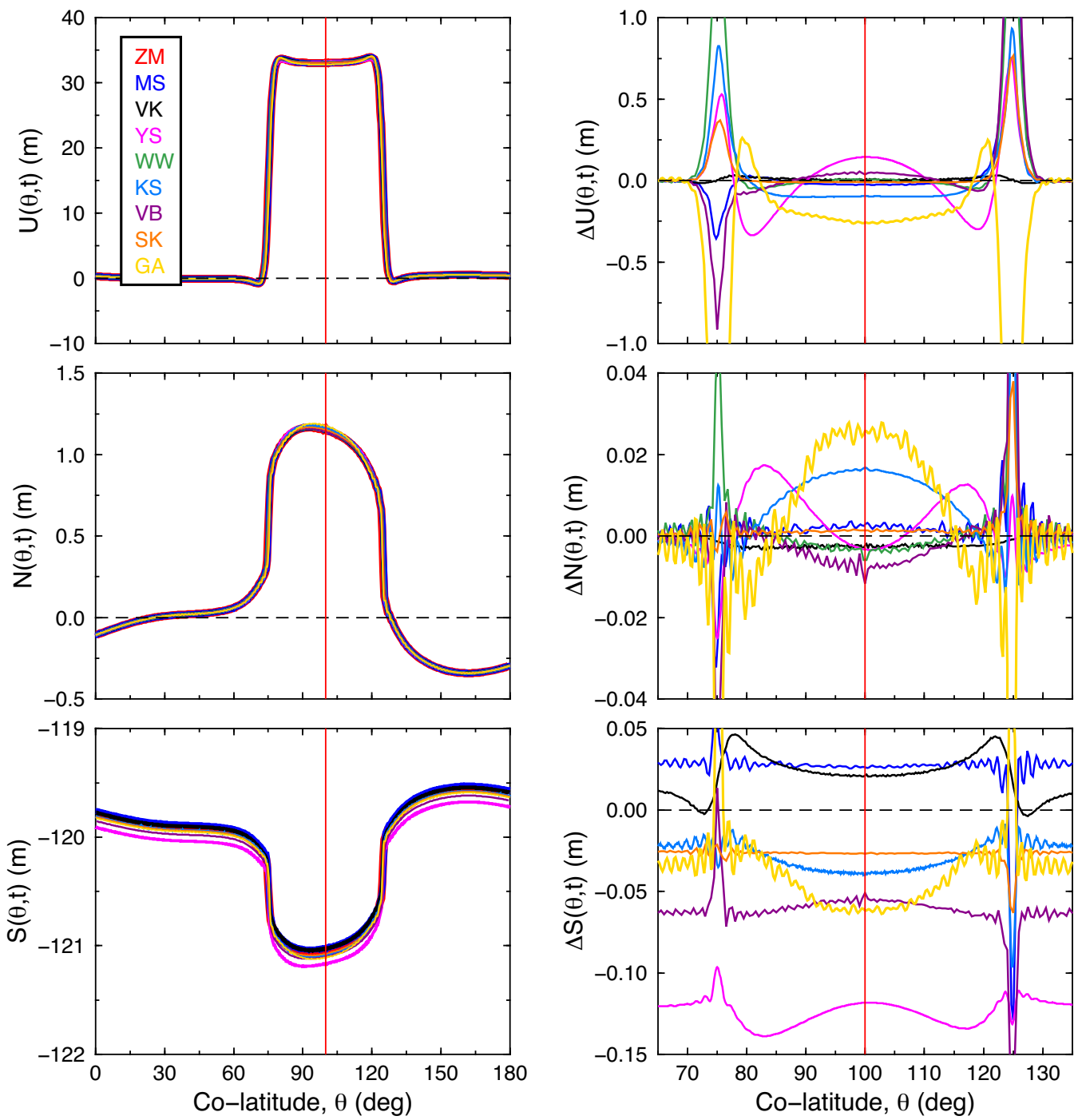

Figure 6. Left-hand panels: the latitudinal cross-section of surface vertical displacement $U$, geoid displacement $N$ and sea-surface variation $S$ through the centre of the ocean basin located at $\left(100^{\circ}, 320^{\circ}\right)$ and time $t=10 \mathrm{kyr}$ provided by all participants (except for WWa). The abbreviations are shown in Table 5 . Right-hand panels: the differences between the individual solutions and VEGA ${ }^{\text {ref }}$ along the co-latitudinal interval $\left(65^{\circ}, 135^{\circ}\right)$. The root-mean square and maximum differences of the individual solutions are shown in Fig. 21.

vertical and geoid displacements reach their maximum values over the ocean basin since accumulated continental ice is extracted from the ocean basin. The water load in the ocean basin is thus reduced after the initial Heaviside unloading and the basin is uplifted over time due to the viscoelastic relaxation of the earth model. The edge of the ocean basin is at $\vartheta=75.7^{\circ}$. Two forebulges have been formed; one outside the basin at $\vartheta=70.4^{\circ}$, and another one inside the basin at $\vartheta=80.6^{\circ}$. The second pair of forebulges is almost symmetrically distributed with respect to the centre of the basin. The left-hand panels of Fig. 6 again show a very good agreement between the individual solutions.

A detailed comparison is provided by the right-hand panels of Fig. 6, which show the differences of the individual solutions with respect to VEGA ${ }^{\text {ref }}$ along the co-latitudinal interval $\left(65^{\circ}, 135^{\circ}\right)$. The differences have both short- and medium-wavelength features with large spikes at the edges of the ocean basin. In contrast to Figs 3 and 5, the spikes are now caused by the Gibbs phenomenon. The right-hand side of eq. (17) contains the difference $N-U$ multiplied by the ocean function $\mathcal{O}$, which has a jump from 0 to 1 at the coast. Converting the product $(N-U) \mathcal{O}$ into the $\mathrm{SH}$ domain results in the Gibbs phenomenon in the loading function. When the load and the associated Gibbs phenomenon are processed by a numerical code, it translates to a Gibbs phenomenon in the viscoelastic response of an earth model. A similar effect is caused by the second term on the right-hand side of eq. (17). The metrics of the differences are graphically shown in the columns denoted by 'B' in Fig. 21. We can see that eight solutions differ by up to 4 and $0.20 \mathrm{~m}$ in the vertical and geoid displacements, with the root-mean square differences reaching up to 0.8 and $0.03 \mathrm{~m}$, respectively. Comparing the RMS along the two profiles, the MS, VK and SK solutions show a larger RMS along the ocean profile, whereas the YS, WW, KS and GA solutions show a larger RMS along the ice profile, all with respect to VEGA $^{\text {ref }}$. 


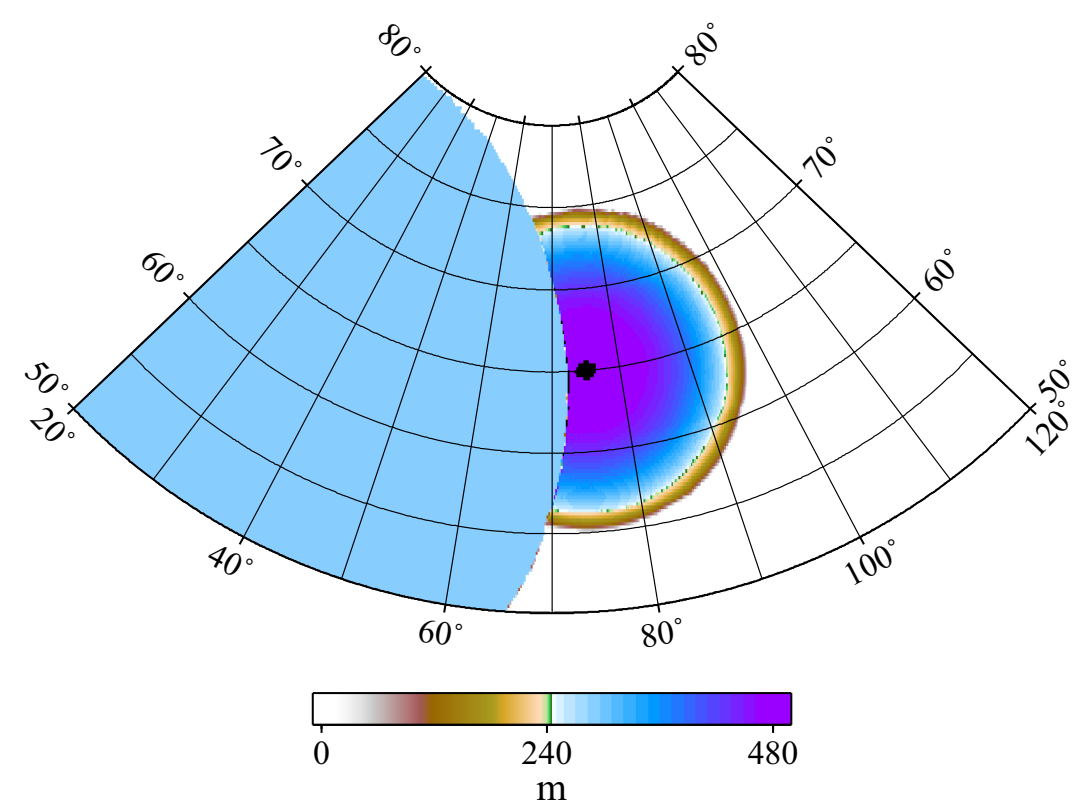

Figure 7. Height of the grounded ice at $t=15 \mathrm{kyr}$ for benchmark C computed by VEGA ${ }^{\text {ref }}$. The grounded ice is calved when it reaches the coast. The light blue sector of the circle shows the ocean function, which is fixed throughout time.

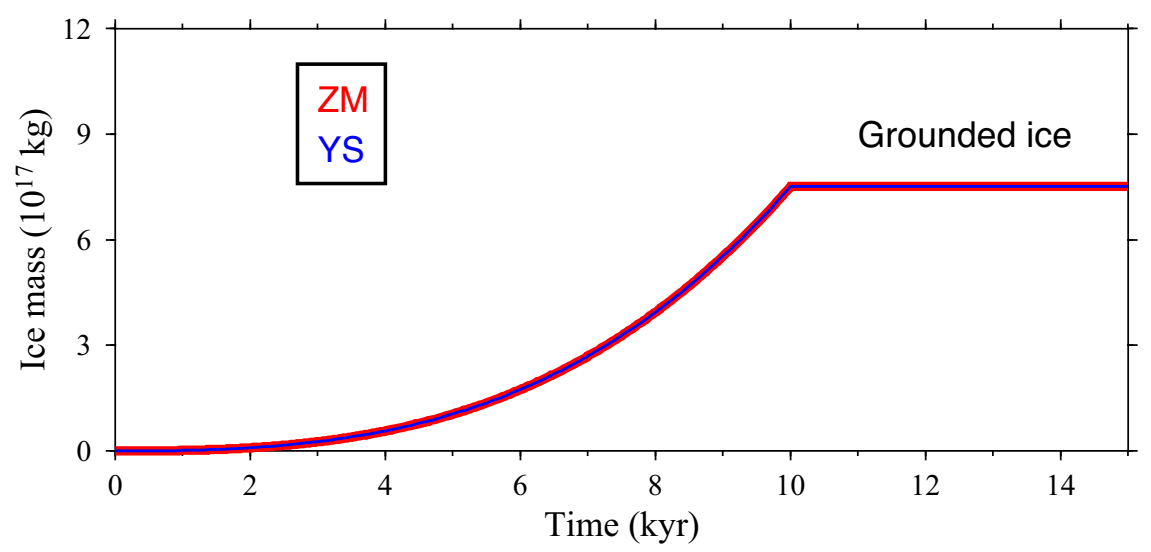

Figure 8. Time evolution of grounded ice for benchmark C.

The bottom right-hand panel of Fig. 6 shows the differences in sea-surface variation $S$. We can see that they are rather systematic, which is likely due to differences in computing $h_{\mathrm{UF}}(t)$; the bias in $h_{\mathrm{UF}}(t)$ reaches $0.12 \mathrm{~m}$.

\subsection{Benchmark C: fixed coastlines, marine-terminating ice sheet}

In benchmark $\mathrm{C}$, the ice is placed near the ocean basin such that it reaches the coastline as it grows over time. The SLE is again solved with a fixed ocean geometry, that is, ice is calved when it reaches the coast. This is illustrated in Fig. 7, where the height of the grounded ice at the terminal time $t=15 \mathrm{kyr}$ is shown. The ocean function drawn by the light blue sector of the circle is fixed throughout time. Additionally, Fig. 8 shows the time evolution of the mass of the grounded ice. Its mass grows approximately cubically with time up to $10 \mathrm{kyr}$, and is then kept constant up to $15 \mathrm{kyr}$. We can see that the results of two participants are in excellent agreement, hence indicating a consistency in the formulation of the load.

The left-hand panels of Fig. 9 show the latitudinal cross-section of $U$ and $N$ through the centre of the ice cap at $t=15$ kyr. Comparing it with Fig. 5, we can see that $U$ and $N$ behave similarly to those in benchmark $\mathrm{B}$, but their amplitudes are now significantly smaller. This is because the size of ice cap gradually grows in the first phase of glaciation in contrast to the Heaviside loading history in benchmark B. In addition, the forebulge has collapsed due to the viscoelastic relaxation of the earth model in the second phase of glaciation. The left-hand panels of Fig. 9 again show an excellent agreement between the five contributing solutions.

The differences of the individual solutions with respect to $\mathrm{VEGA}^{\text {ref }}$ along the co-latitudinal interval $\left(5^{\circ}, 45^{\circ}\right)$ are shown in the right-hand panels of Fig. 9. In contrast to benchmark B, the differences have predominantly medium-wavelength features, since most of the shortwavelength differences are dampened by the relaxation of the earth model in the second phase of glaciation. While the differences for the 
Benchmark $\mathrm{C}\left(\lambda=75^{\circ}, \mathrm{t}=15 \mathrm{kyr}\right)$
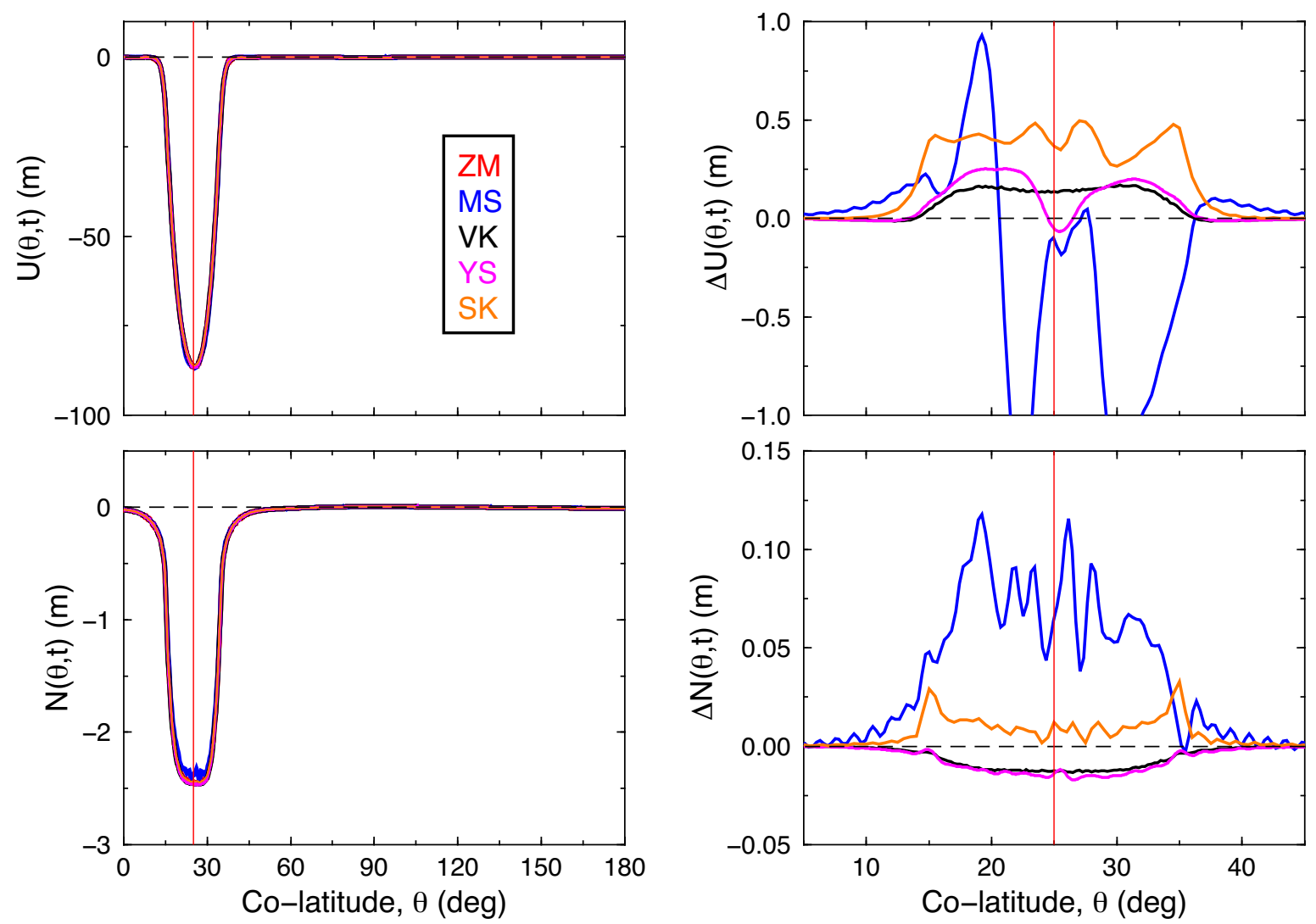

Figure 9. The same as Fig. 5, but for benchmark $\mathrm{C}$ and the terminal time of glaciation at $15 \mathrm{kyr}$.

VK, YS and SK solutions are similar to those for benchmark B, the differences for the MS solution are now larger compared to the previous case. This is also documented by the difference metrics in the third columns of the panels in Fig. 20.

Fig. 10 shows the latitudinal cross-section of $U, N$ and $S$ through the centre of the ocean basin at $t=15$ kyr. The interpretation of the left-hand panels of Fig. 10 are similar to those in Fig. 6 for benchmark B with reduced amplitudes of $U, N$ and forebulges in $U$. The differences of the individual solutions with respect to $\mathrm{VEGA}^{\text {ref }}$ along the co-latitudinal interval $\left(0^{\circ}, 70^{\circ}\right)$ shown in the right-hand panels of Fig. 10 have predominantly medium-wavelength features as in Fig. 9. The metrics of the differences are graphically shown in the columns denoted by 'C' in Fig. 21. We can see that the differences between the solutions are significantly smaller than those for benchmark B, which is probably caused by the smaller amplitudes of $U$ and $N$ for benchmark C compared to benchmark B. The differences in sea-surface variation $S$ shown in the bottom right-hand panel of Fig. 10 are again systematic as in Fig. 6, indicating a bias of $0.2 \mathrm{~m}$ in calculating $h_{\mathrm{UF}}(t)$.

In addition, Fig. 11 shows the time evolution of the thickness of the uniform water layer, $h_{\mathrm{UF}}(t)$. Blue and red curves stand for two terms on the right-hand side of eq. (12), while the sum of them is shown by the black curve. The blue curve thus represents the mass extracted from the ocean basin that is used to build the continental ice. This curve exactly anticorrelates with the grounded ice-mass growth shown in Fig. 8. The red curve represents the thickness of the uniform water layer due to the difference between the ocean-bottom displacement $U$ and the geoid displacements $N$. The positive sign of this component can be attributed to the dominating uplift of the ocean bottom (see the top left-hand panel in Fig. 10) compared to the geoid displacement $N$ (see the middle left-hand panel of Fig. 10), which is caused by the water-mass loss of the ocean basin.

\subsection{Benchmark D: moving coastlines, floating ice}

The setup of benchmark D is the same as that of benchmark C, but the SLE is now solved with a time-varying ocean geometry and floating ice. This means that when the advancing ice reaches the edge of the ocean basin, it continues as grounded ice if $h_{\mathrm{w}}^{*}(\Omega, t) \leq 0$, where $h_{\mathrm{w}}^{*}(\Omega, t)$ is given by eq. (22); otherwise, it floats. This is illustrated in Fig. 12, where the height of grounded ice at the terminal time $t=15 \mathrm{kyr}$ is shown. The ocean function, which now varies over time, is shown by the light blue sector of circle. The comparison of Fig. 12 with Fig. 7 shows the grounded ice now extending into the ocean basin. Fig. 13 shows the time evolution of the grounded and floating ice. The comparison of this figure with Fig. 8 shows the mass of the grounded ice is now larger than for benchmark $\mathrm{C}$, since the grounded ice extends in the ocean basin while it is calved at the coast in benchmark $\mathrm{C}$. The plotted results of four participants are in good agreement. 
Benchmark $C\left(\lambda=25^{\circ}, \mathrm{t}=15 \mathrm{kyr}\right)$
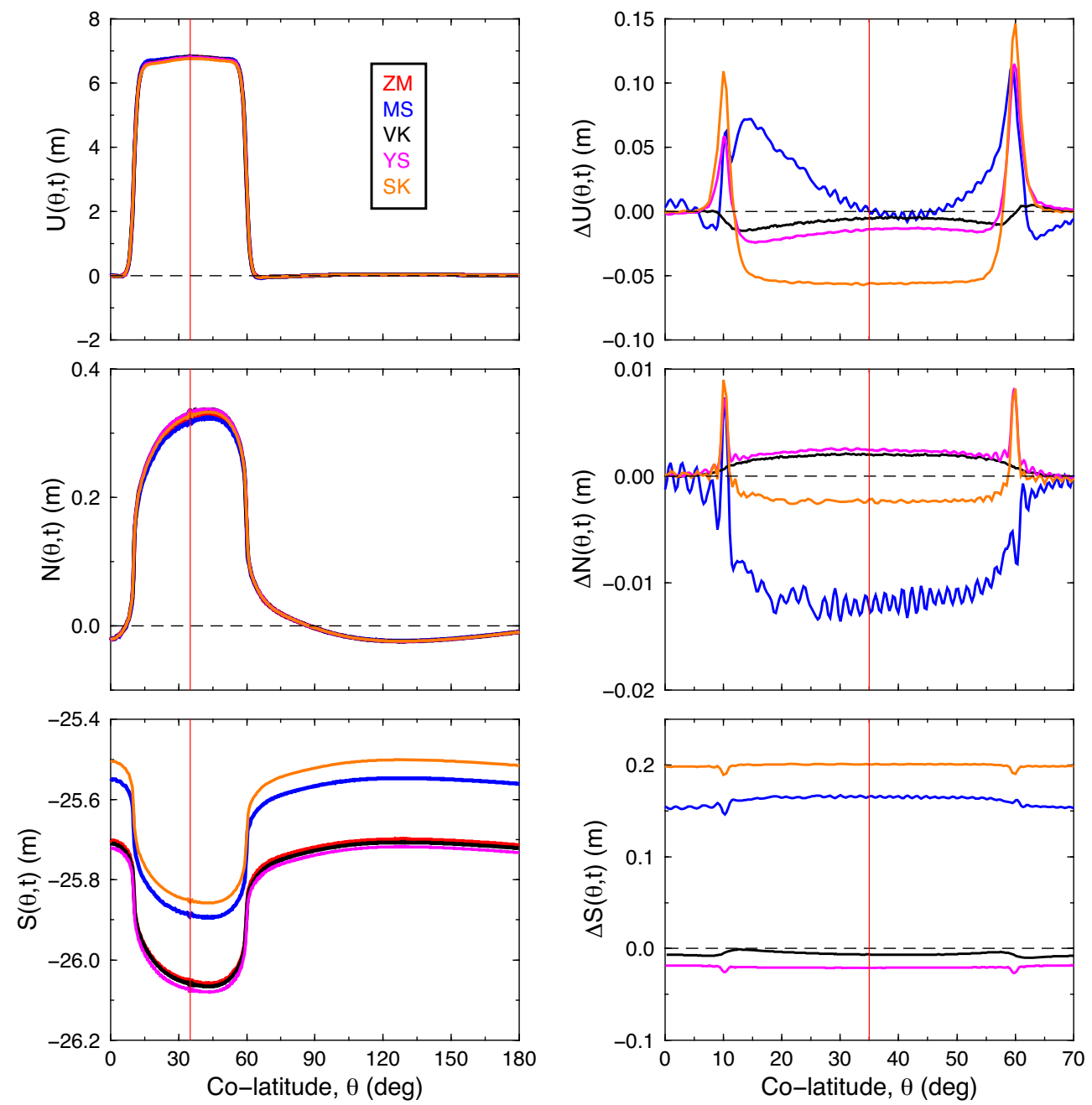

Figure 10. The same as Fig. 6, but for benchmark C. The centre of the ocean basin is located at $\left(35^{\circ}, 25^{\circ}\right)$, which is indicated by the red vertical line.

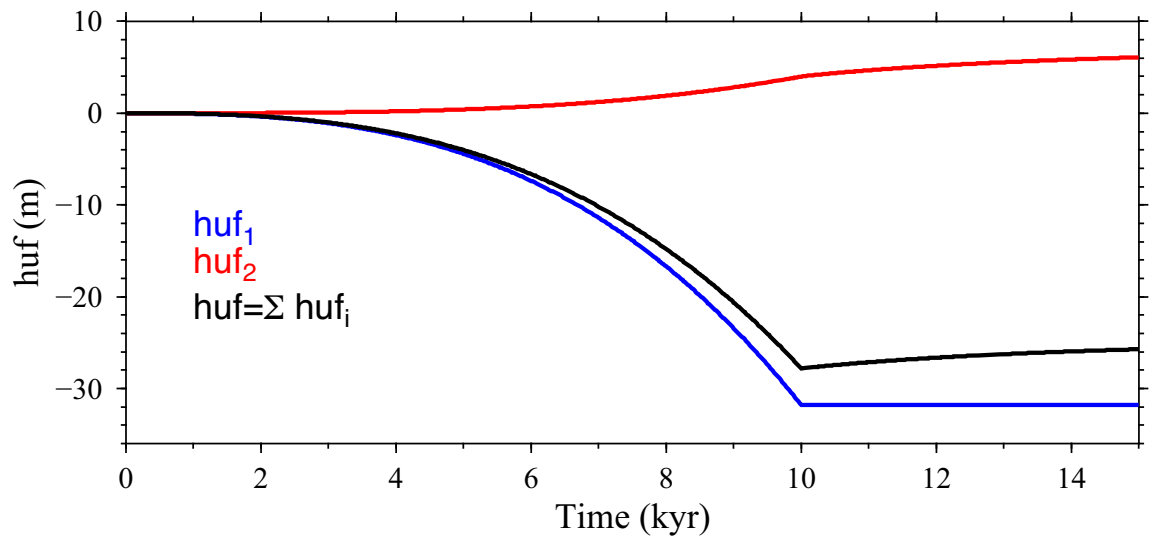

Figure 11. Time evolution of the thickness of the uniform water layer, $h_{\mathrm{UF}}(t)$, for benchmark $\mathrm{C}$ computed by VEGA ${ }^{\text {ref }}$. The blue and red curves stand for two terms on the right-hand side of eq. (12), while the sum of them is shown by the black curve.

The left-hand panels of Figs 14 and 15 show the latitudinal cross-sections of $U$ and $N$ through the centre of the ice load and the centre of the ocean basin, respectively, at $t=15 \mathrm{kyr}$. The comparison of these figures with Figs 9 and 10 shows that $U$ and $N$ behave similarly as those in benchmark $\mathrm{C}$, but their amplitudes are now about 10 per cent larger. This is because the ice advancing to the ocean basin was calved at the coast of the ocean basin in the previous case and thus it did not form a load on the ocean shelf, while now the ice continues to advance (up to a certain distance from the coast) into the ocean basin as grounded ice. Hence, the ocean shelf is now loaded by grounded ice. 


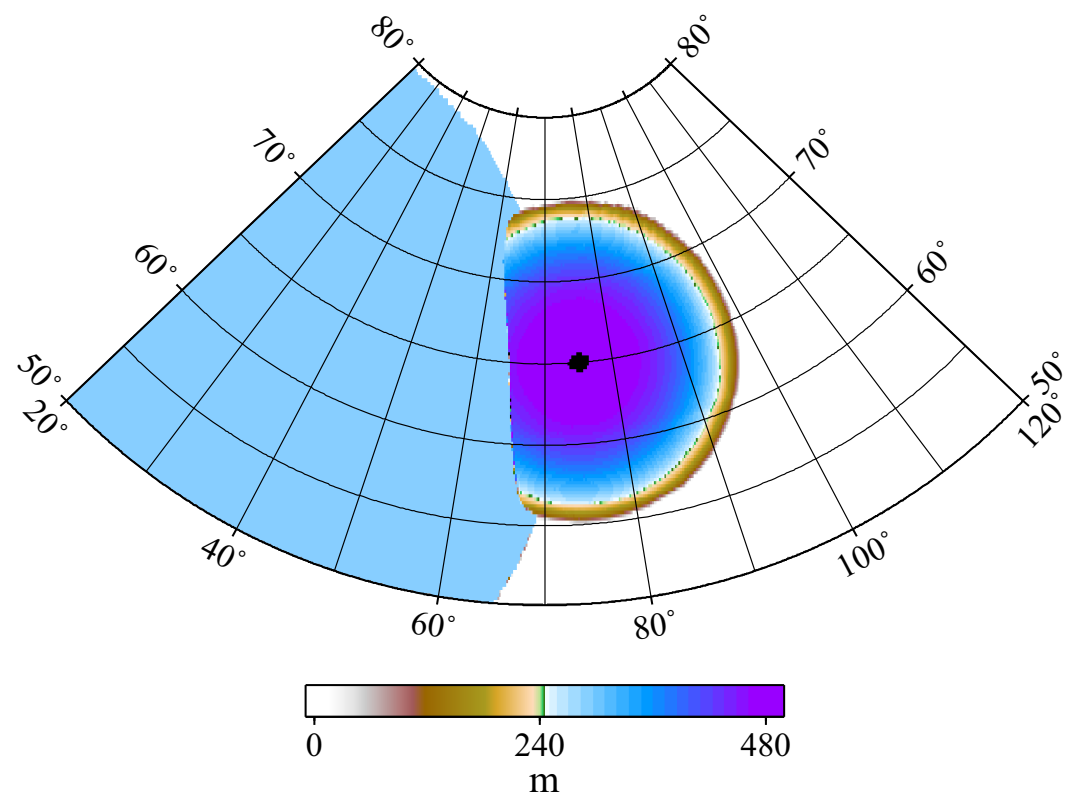

Figure 12. Height of the grounded ice at $t=15 \mathrm{kyr}$ in benchmark D computed by VEGA ${ }^{\text {ref }}$. The light blue sector of the circle shows the ocean function, which varies over time.

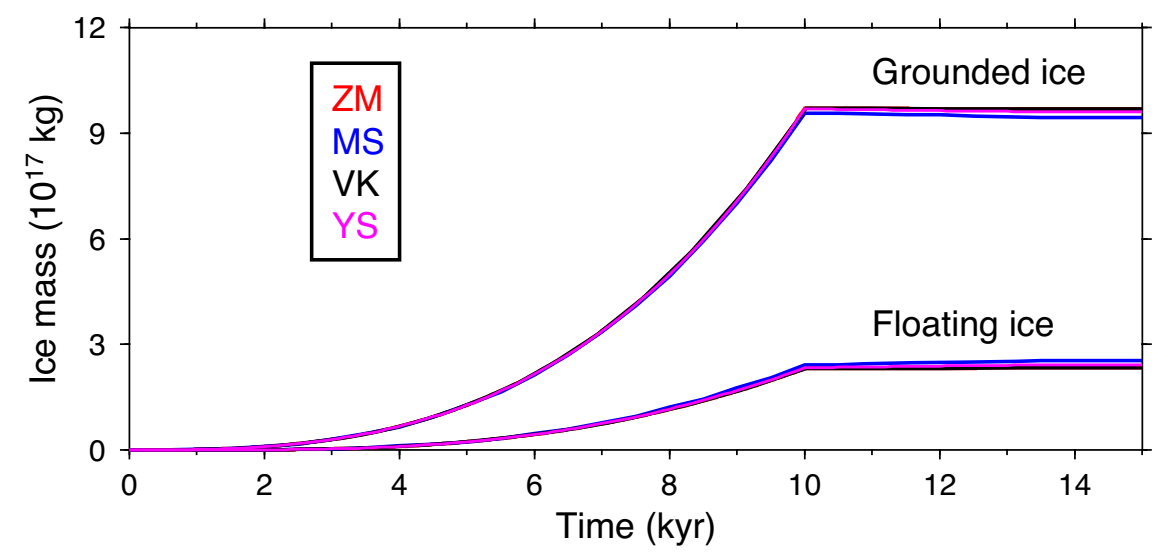

Figure 13. Time evolution of grounded and floating ice for benchmark D.

The results of five participants of the benchmark study are available for benchmark D. Their differences with respect to VEGA ${ }^{\text {ref }}$ along the co-latitudinal intervals $\left(5^{\circ}, 45^{\circ}\right)$ and $\left(0^{\circ}, 70^{\circ}\right)$ are shown in the right-hand panels of Figs 14 and 15 , respectively. The differences for the VK and YS solutions have a similar feature as those for benchmark $\mathrm{C}$, with similar difference metrics as shown in the fourth columns of Figs 20 and 21. The differences of the MS solution also partly resemble those for benchmark C. In all cases, it is important that the behaviour of the differences and their amplitudes do not change significantly when the fixed ocean geometry (benchmark C) in the SLE is replaced by a time-varying ocean geometry and floating ice (benchmark D).

Fig. 16 shows the time evolution of the thickness of the uniform water layer, $h_{\mathrm{UF}}(t)$. Blue, red and yellow curves stand for the three terms on the right-hand side of eq. (19), while the sum of them is shown by the black curve. The blue and red contributions have the same meaning as those in Fig. 11. The yellow curve represents the water mass change due to moving coastlines. The insert in Fig. 16 shows the very good agreement between the individual solutions (except for SK).

Finally, Fig. 17 shows the time evolution of the area of the ocean basin as determined by five participants, that is, the area of the time-varying ocean function $\mathcal{O}(\Omega, t)$ on a unit sphere, which is given by $\overline{\mathcal{O}}(t)$ in eq. (21). As expected, the area of the ocean basin shrinks over time, with a reasonable agreement between the different results.

\subsection{Benchmark E: moving coastlines, floating ice, terminal SET}

When modelling GIA, we are faced with the case that the SET and other boundary data need to be specified at some terminal time $t_{\mathrm{f}}$ and the solution of GIA governing equations is desired for $t<t_{\mathrm{f}}$. However, the GIA is traditionally described by a Maxwell viscoelastic 
Benchmark $D\left(\lambda=75^{\circ}, t=15 \mathrm{kyr}\right)$
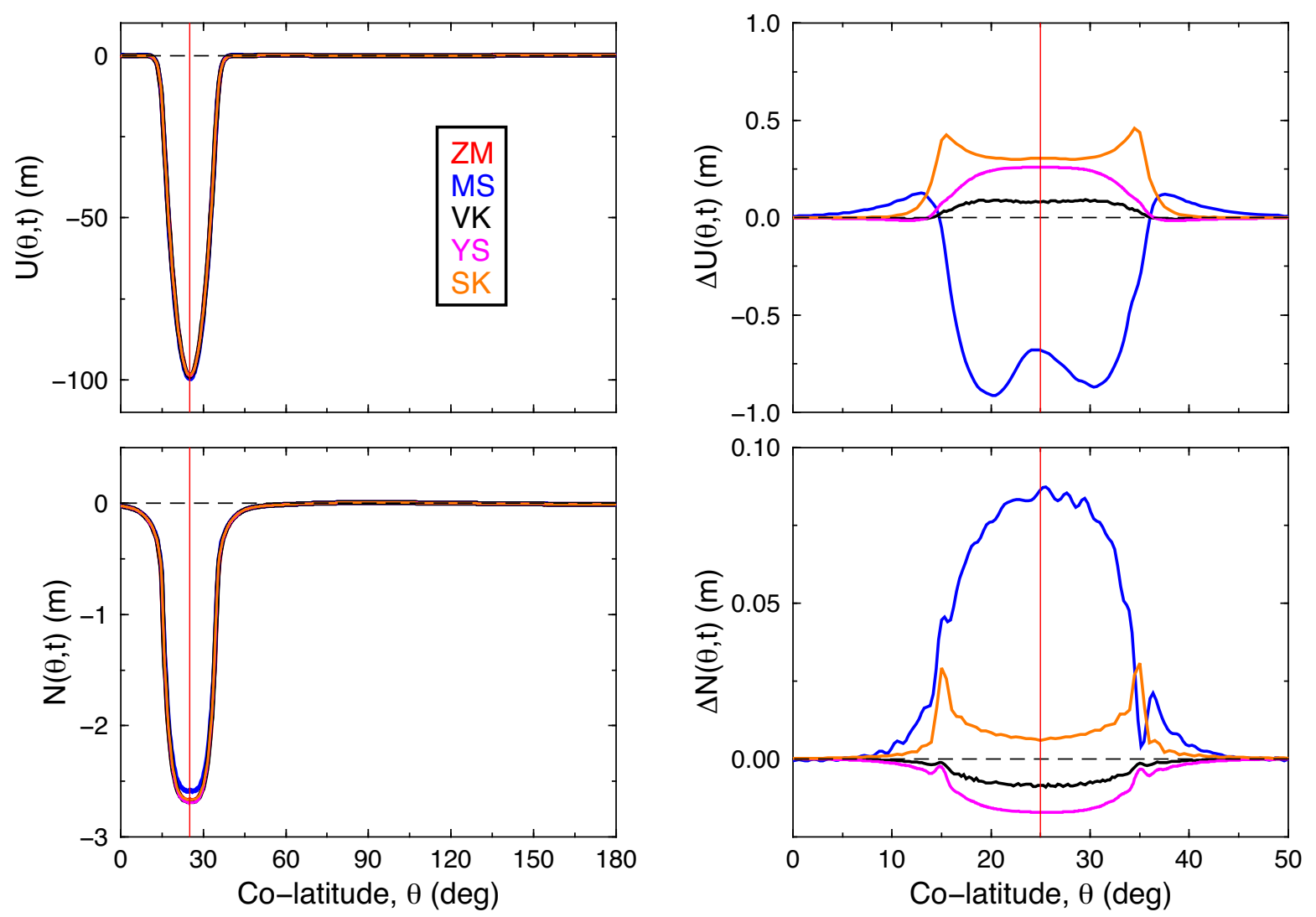

Figure 14. The same as Fig. 9, but for benchmark D.

relaxation which is a diffusion process in time that makes initial conditions, that is, an initial surface-mass load distribution and initial SET, smoother. The change of variables $t \rightarrow-t$ converts a viscoelastic diffusion process to a problem with negative diffusion. While the forward-in-time viscoelastic process makes the initial conditions smoother, the backward-in-time viscoelastic process makes the terminal conditions rougher. In mathematical terminology, a forward-in-time viscoelastic process is a well-posed initial problem in the Hadamard sense, while a backward-in-time viscoelastic process is an ill-posed problem in a sense that a high-frequency content in the initial data is amplified when the viscoelastic process is solved backward-in-time. A physical explanation provides the second law of thermodynamics stating that a diffusion process is irreversible in time since its entropy production increases in time. The following test example shows how to deal with the case when the SET is specified at terminal time $t_{\mathrm{f}}$. It should be noted that erosion and sediment deposition is not considered here.

The setup of benchmark E is the same as benchmark D, but instead of prescribing an initial SET in benchmark D, its geometry at the terminal time is defined as the input parameter for benchmark E. The initial, pre-glacial SET is now searched for such that its desired geometry at the terminal time $\zeta^{(t)}$, des is adjusted. We choose $\zeta^{(t) \text {, des }}$ in the form of eq. (29), that is,

$\zeta^{(t), \operatorname{des}}(\Omega)=b_{\max }-b_{0} \exp \left\{-\psi^{2} / 2 \sigma_{b}^{2}\right\}$

where the parameters $b_{\max }$ and $b_{0}$ are those used in benchmark D.

As defined by eq. (3), the sea level height $h_{\mathrm{w}}(\Omega, t)$ is the ocean water thickness at the point $\Omega$ and time $t$. The SET at the terminal time $t_{f}$, $\zeta^{(t)}(\Omega)$, is then the negative $h_{\mathrm{w}}\left(\Omega, t_{f}\right)$ at the point $\Omega$ and time $t_{f}$. In view of eq. (15), the relationship between the terminal and initial SETs is

$\zeta^{(t)}(\Omega)=\zeta^{(0)}(\Omega)+U(\Omega, t)-N(\Omega, t)-h_{\mathrm{UF}}(t)$

Since the time-varying ocean geometry depends on $\zeta^{(0)}$, the deformation response of the Earth to the surface loading by the SLE depends non-linearly on $\zeta^{(0)}$. Hence, eq. (33) cannot be solved directly for $\zeta^{(0)}$ by assuming that all other variables are known, but an iterative approach must be carried out. Moreover, the second term on the right-hand side of eq. (17) shows that the influence of the initial SET becomes important only for locations near the coast. Hence, the nonlinearity of the GIA solution with respect to $\zeta^{(0)}$ is rather weak, and an iterative approach for searching $\zeta^{(0)}$ may be relatively simple to implement. 
Benchmark D $\left(\lambda=25^{\circ}, \mathrm{t}=15 \mathrm{kyr}\right)$
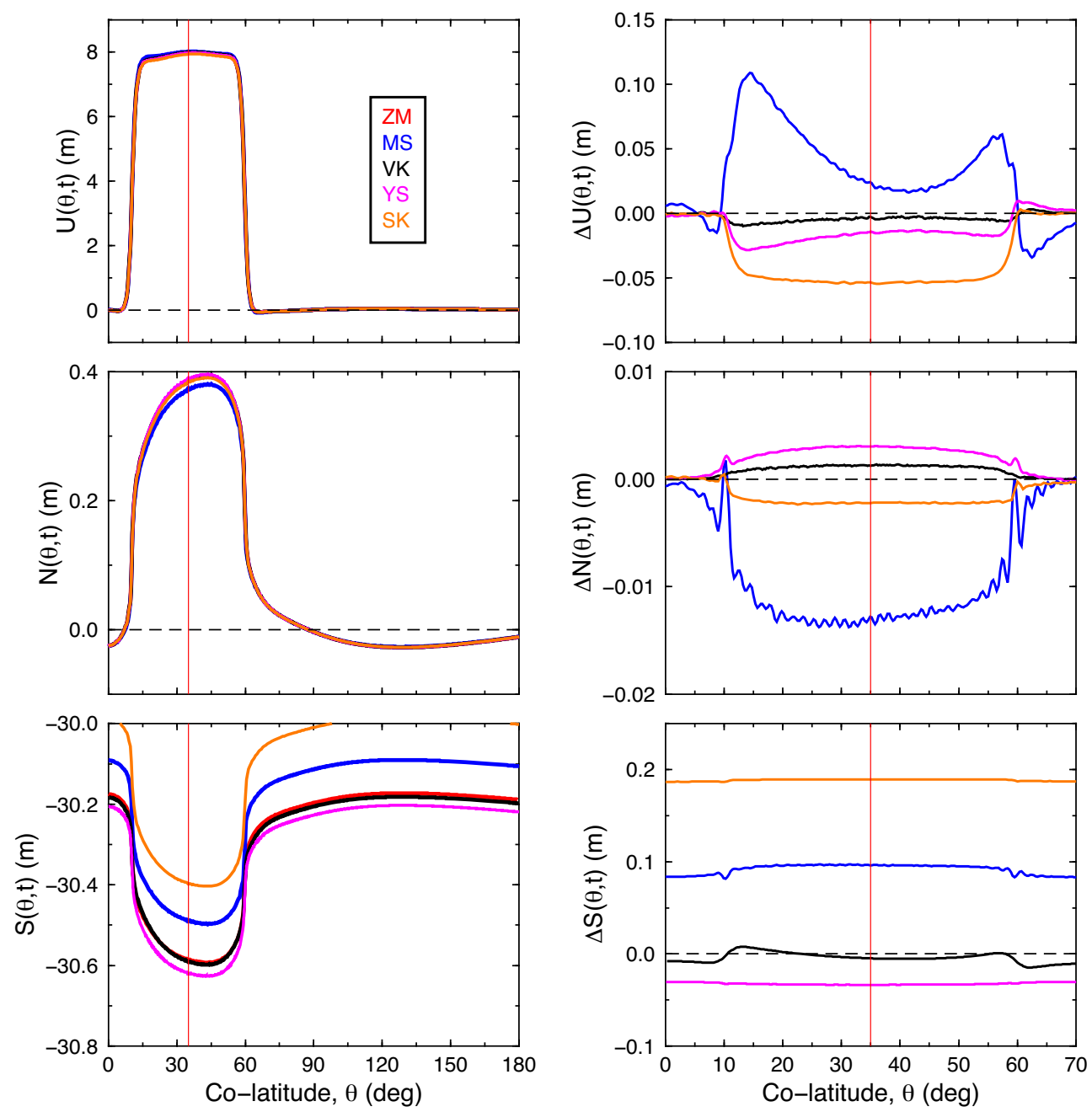

Figure 15. The same as Fig. 10, but for benchmark D.

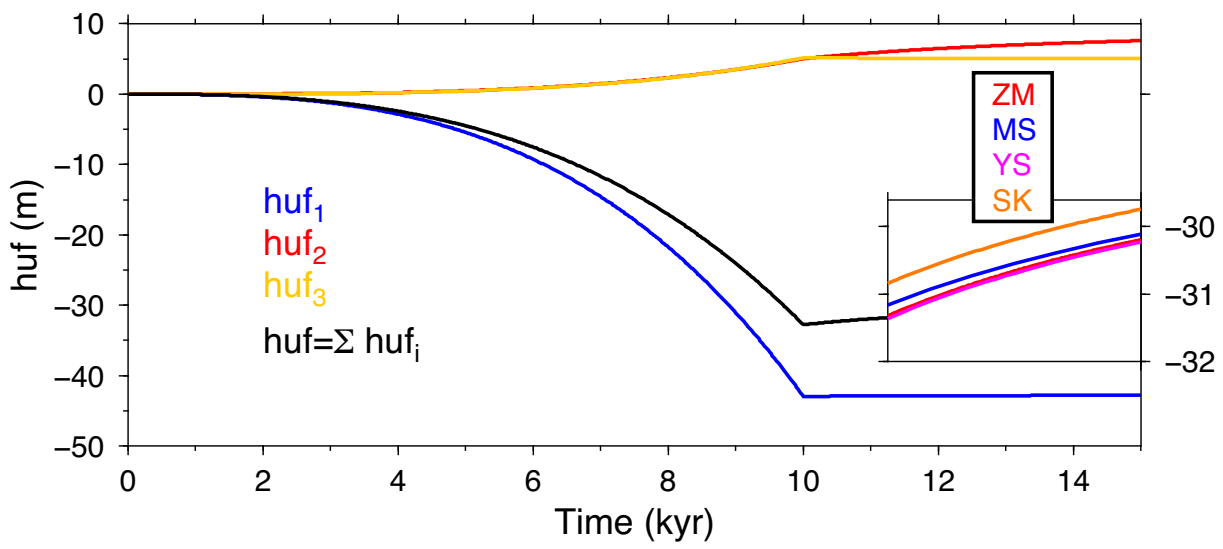

Figure 16. Time evolution of the thickness of the uniform water layer, $h_{\mathrm{UF}}(t)$, for benchmark D. Blue, red and yellow curves stand for three terms on the right-hand side of eq. (19), while the sum of them is shown by the black curve. The results of four participants are expanded in the insert.

One way is as follows. We begin with an estimate of the initial SET, $\zeta^{(0), 0}$, compute the GIA solution and find the terminal SET, $\zeta^{(t), 0}$, according to eq. (33). Then we consider the sequence $\zeta^{(0), 1}, \zeta^{(0), 2}, \ldots$, such that

$\zeta^{(0), k+1}=\zeta^{(0), k}+\zeta^{(t), \text { des }}-\zeta^{(t), k}$, 


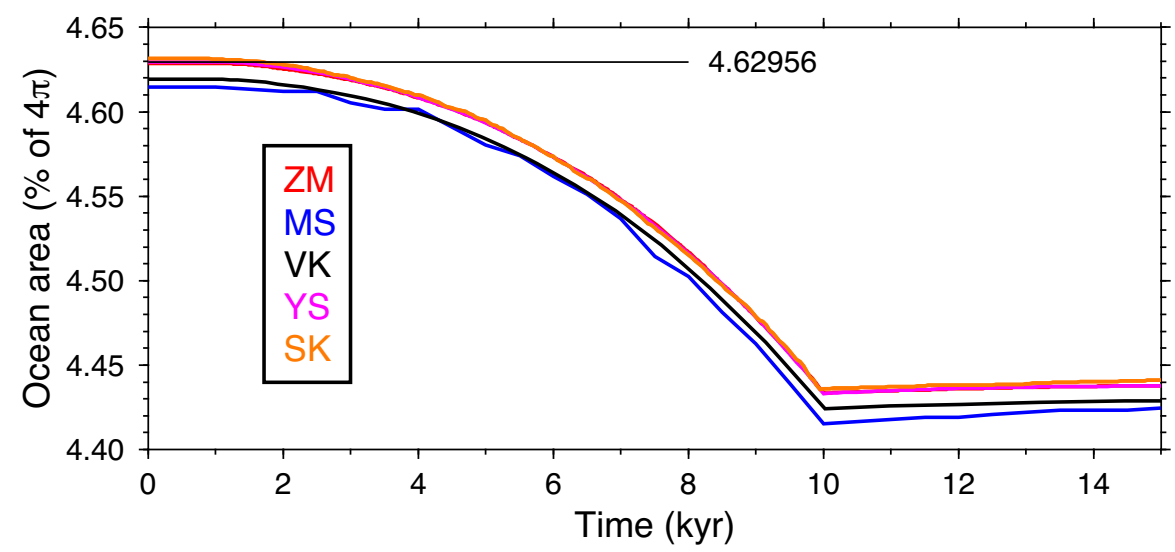

Figure 17. Time evolution of the area of the ocean basin in benchmark D. The radius of the ocean basin at $t=t_{0}$ is $\alpha_{0}=24.8503^{\circ}$ with the area of $A / 4 \pi=(1$ $\left.-\cos \alpha_{0}\right) / 2=0.0462956$. The results of five participants are plotted.

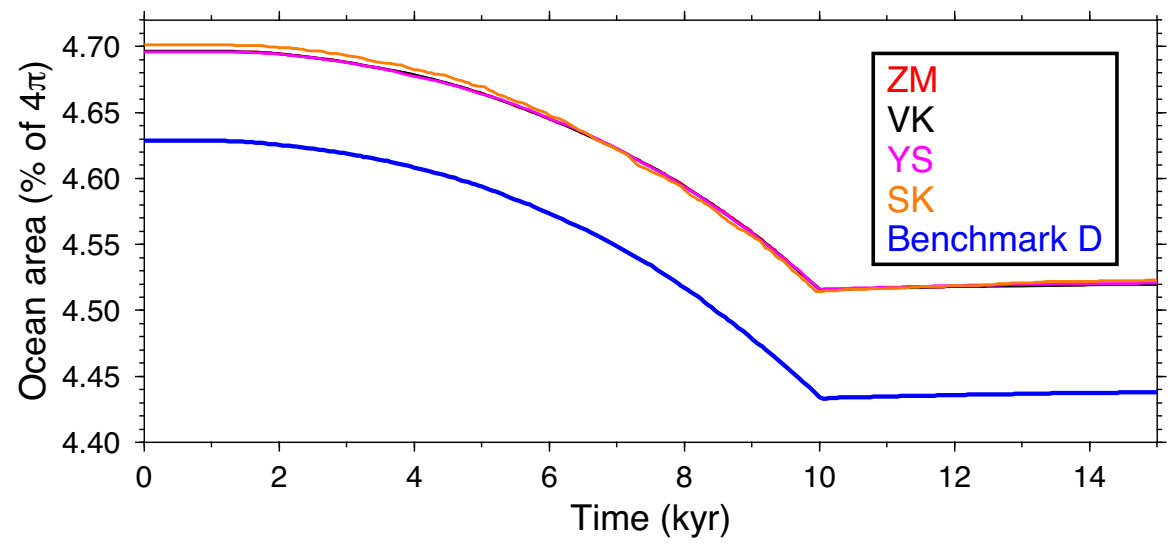

Figure 18. Time evolution of the area of the ocean basin in benchmark E. The results of four participants are plotted and compared with the same quantity for benchmark D.

where the desired terminal SET is in our case given by eq. (32). The iterations continue until the L2 norm of the difference $\zeta^{(t) \text {, des }}-\zeta^{(t), k}$ is smaller than a prescribed tolerance of $0.01 \mathrm{~m}$. This results in a maximum of six iterations.

The area of the ocean basin, that is, the average $\overline{\mathcal{O}}(t)$ given by eq. (21), is a possible measure to compare the numerical results of participants. Fig. 18 shows $\overline{\mathcal{O}}(t)$ as a function of time for four individual solutions. For comparison, the same quantity is plotted for benchmark D. We can make two conclusions. First, the area of the ocean basin is greater for benchmark E than for benchmark D. This is due to the fact that the bottom of the ocean basin is lifted up during the GIA relaxation process because of the extraction of water from the basin for building the continental grounded ice. Hence, prescribing the terminal SET in benchmark E in the same form as the initial SET in benchmark D implies that the ocean basin in benchmark E is deeper than in benchmark D. Due to the form of eq. (29), it also means that the area of the ocean basin is greater for benchmark E than for benchmark D. Second, the results by ZM, VK, YS and SK are in excellent agreement.

The thickness of the uniform water layer, $h_{\mathrm{UF}}(t)$, is an alternative integral measure used to compare the numerical results of the participants. Fig. 19 shows the time evolution of $h_{\mathrm{UF}}(t)$ for the solutions by ZM, VK, YS and SK. For comparison, the same quantity is plotted for benchmark D. We can see that less water is extracted from the ocean basin for benchmark E than for benchmark D. The results by ZM, VK and YS are again in excellent agreement and coincide with that of benchmark D, while those by SK differ from the other solutions. We associate these differences with an inaccuracy in computing $h_{\mathrm{UF}}(t)$ by the SK approach. This can be seen in Figs 10 and 15 , where the sea-surface variation for the SK solution differs systematically by $0.2 \mathrm{~m}$ from the ZM, VK and YS solutions.

\section{CONCLUSIONS}

This paper has been motivated by an effort to extend the Spada et al.'s (2011) benchmark study of various GIA modelling codes (and mathematical formulations) that incorporate the SLE in GIA modelling. Such a comparison is absent from the earlier study. We have designed a set of five synthetic examples described in Sections 3.1-3.5. The benchmark examples range from simple to moderate complexity because to achieve an agreement for case studies of high complexity is, according to our experience, very difficult or even impossible (e.g. Souček \& Martinec 2011). The examples proposed here will, however, serve as the basis for other benchmark studies, such as the solution of the 


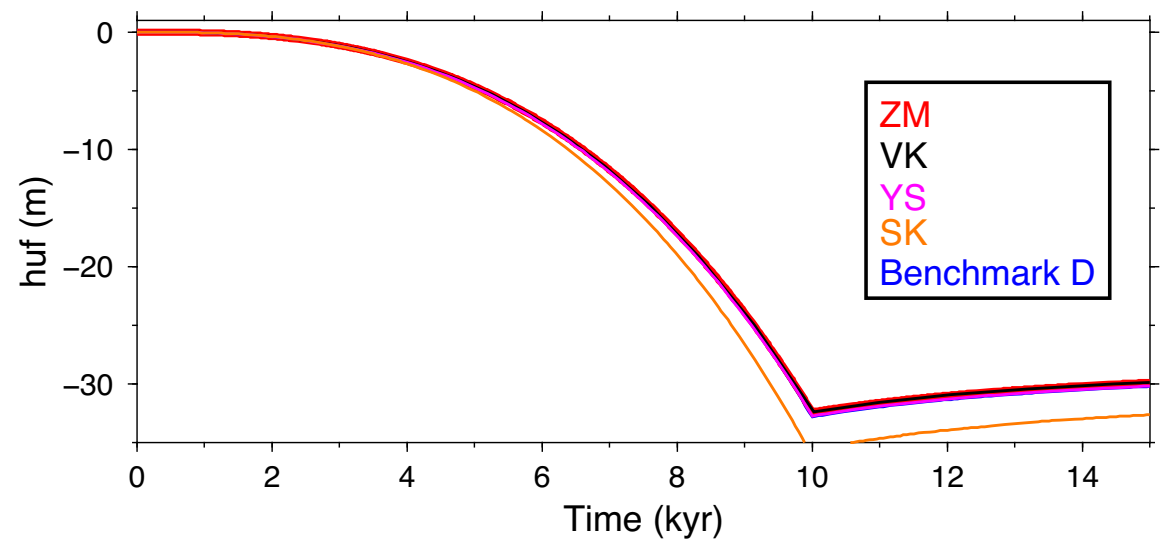

Figure 19. Time evolution of the thickness of the uniform water layer, $h_{\mathrm{UF}}(t)$, for benchmark E. The results of four participants are plotted and compared with the same quantity for benchmark D.
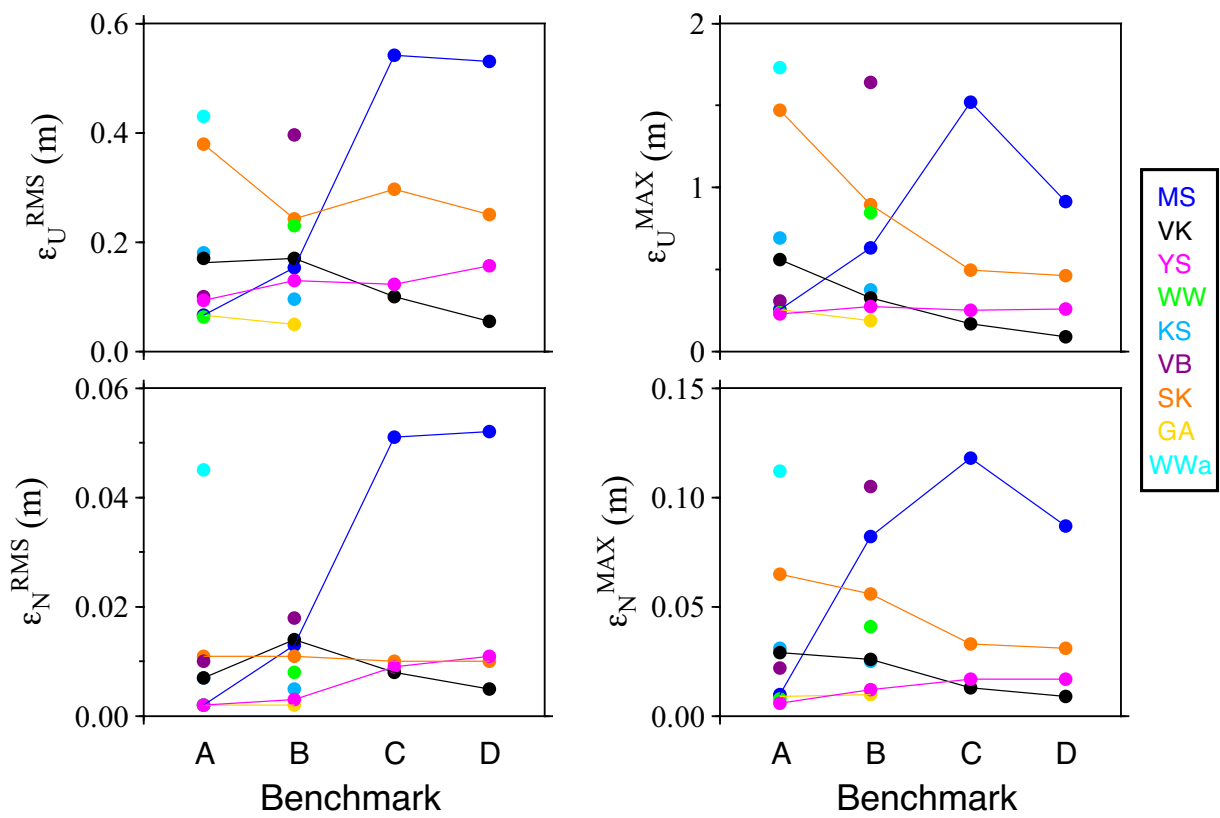

Figure 20. The root-mean square and maximum differences of the individual solutions for $U$ and $N$ along the co-latitudinal cross-sections through the centre of the ice cap, which are shown in the right-hand panels of Figs 3, 5,9 and 14 for benchmark examples A-D. The difference metrics are defined by eq. (31).

GIA model equations with a realistic loading scenario, solutions with rotational feedback in the linear-momentum equation (Martinec \& Hagedoorn 2014) and considering a 3-D viscosity structure of the Earth's mantle.

The suite of codes that are tested represents a combination of different numerical methods of time and spatial parametrizations. Two different parametrizations, the explicit time-domain discretization and the viscoelastic surface load Love numbers computed in the Laplace domain, are used to evolve the SLE over time, while the SH-FD, SH-FE and 3D FE methods (see Table 5) are used for the spatial parametrization of GIA field variables. The surface ice-water load and solid Earth's topography are represented in the spatial domain on the grid of the GaussLegendre latitudinal nodes and equiangle longitudinal nodes (VEGA, VILMA and MUSSEL), the equiarea, icosahedron-shaped, spherical pixels (SELEN), and equiangular longitudinal and latitudinal nodes (TUDSLE, PGCcalc, giapy, TSec02 and A13).

The tested codes differ in the degree of the complexity of SLE implementation. The AFCAL code does not include the SLE in the boundary condition for GIA modelling, four codes (TUDSLE, TSec02, PGCcalc and A13) consider the SLE for a fixed time-ocean geometry, while five codes (VEGA, VILMA, SELEN, MUSSEL and giapy) implement a full form of the SLE with a time-varying ocean geometry. The intercomparison between the numerical results of the codes shows that none of them produces significantly different results. We showed that the differences in surface vertical displacement and geoid displacement do not exceed 1.5 and $0.1 \mathrm{~m}$ (which represents around 1.5 per cent of the response) with root-mean squares of 0.5 and $0.02 \mathrm{~m}$, respectively. The main source of the differences is due to different spatial discretizations of the SLE and field variables, which consequently results in different treatments of the Gibbs oscillatory behaviour of solutions at the margin of the ice cap and ocean basin. The plots of sea-surface variation $S$ show that the individual solutions differ rather systematically, which is caused by the differences in computing $h_{\mathrm{UF}}(t)$ with the bias reaching $0.2 \mathrm{~m}$. 

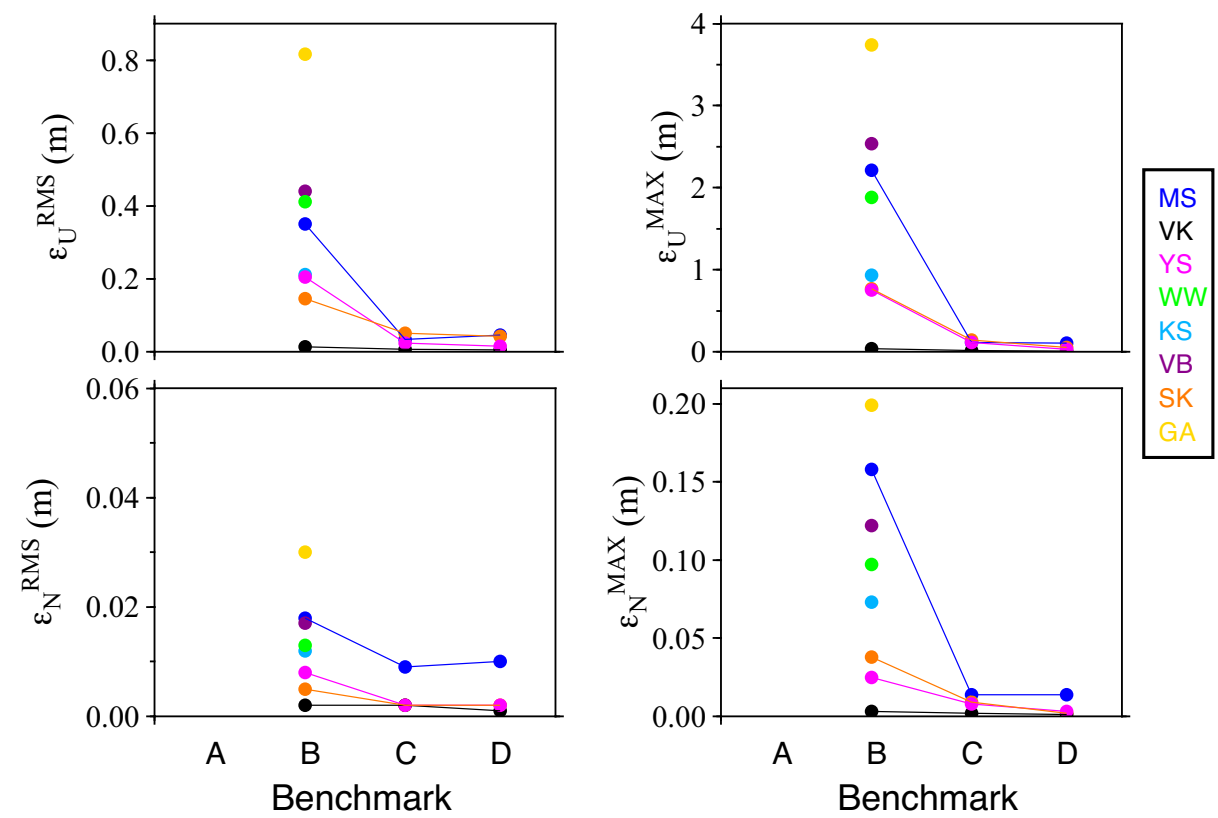

Figure 21. The same as Fig. 20, but for the co-latitudinal cross-sections through the centre of the ocean basin, which are shown in the left-hand panels of Figs 6,10 and 15 for benchmark examples B-D. The results for benchmark A are not applicable since an ocean basin is not considered in this case.

A minor source of difference arises from the different approaches to the time-implicit character of the SLE, which is tackled in this benchmark by two different ways. Four time-domain based codes (VEGA, VILMA, AFCAL and giapy) solve the SLE pointwise on a spatial grid by an explicit time-differencing scheme with the same time step as for the viscoelastic field equations. The six Laplace-domain based codes (SELEN, TUDSLE, PGCcalc, MUSSEL, TSec02 and A13) solve the SLE iteratively over time by assuming a piecewise constant behaviour of all field variables involved in the SLE, with a typical time step between 0.1 and $1 \mathrm{kyr}$. The time iterations usually require a maximum of six steps, which ensures that the relative precision of the solution is better than 1 per cent.

We can conclude that, even though the tested numerical implementations of the SLE are very different in their numerical approaches, all benchmark results are reasonably consistent with each other, suggesting that these solutions are all equally valid in approaching GIA modelling with the SLE as a free boundary-value function. The benchmark examples show the accuracy that can be expected from different implementations of the SLE, which helps to assess differences in the literature between predictions for realistic loading cases.

We believe that the results presented in this study will be of benefit to the GIA community and, specifically, for scientists approaching the modelling of GIA for the first time. This is in line with the rationale of the previous GIA-oriented benchmark study (Spada et al. 2011). Moreover, the work presented here has provided a number of benefits for the scientists involved. In fact, during the various stages of the benchmark activities, the participants had the opportunity of addressing previous misunderstandings about the SLE theory, correcting mistakes in the numerical implementation of the theory, and improving existing numerical algorithms.

\section{ACKNOWLEDGEMENTS}

The authors would like to thank Kevin Fleming, Patrick Wu and Anthony Purcell for their comments that have helped to improve the manuscript. This research was supported by the Grant Agency of the Czech Republic, project No. P210/17-03689S, and by the research program 11/RFP.1/GEO/3309 financed by the Science Foundation of Ireland. ZM acknowledges this support. VK was supported by the German Federal Ministry of Education and Research under the Research for Sustainability Initiative through Palmod project FKZ: 01LP1503A. GS was supported by a DiSPeA research grant (CUP H32I160000000005). RR acknowledges funding by the Netherlands Organization for Scientific Research, through VIDI Grant No. 864.12.012. YS acknowledges funding by the TU Delft Climate Institute. VB acknowledges funding by the European Space Agency in the framework of the Sea Level Budget Closure CCI Project as a part of ESA's Climate Change Initiative. TJ was supported by Lands and Minerals Sector (Natural Resources Canada) contribution number 20180013.

\section{REFERENCES}

A, G., Wahr, J. \& Zhong, S., 2013. Computations of the viscoelastic response of a 3-D compressible Earth to surface loading: an application to glacial isostatic adjustment in Antarctica and Canada, Geophys. J. Int., 192, 557-572.

Barletta, V.R. \& Bordoni, A., 2013. Effect of different implementations of the same ice history in GIA modeling, J. Geodyn., 71, 65-73.
Barletta, V.R. \& Spada, G., 2012. Assessment of errors and uncertainty patterns in GIA modeling, in Geophysical Research Abstracts, Vol. 14, EGU2012-9717, EGU General Assembly.

Barletta, V.R. et al., 2013. Fingerprinting sea-level variations in response to continental ice loss: a benchmark exercise, in Geophysical Research Abstracts, Vol. 15, EGU2013-8879, EGU General Assembly. 
Farrell, W.E. \& Clark, J.A., 1976. On postglacial sea level, Geophys. J., 46, 79-116.

Gasperini, P. \& Sabadini, R., 1989. Lateral heterogeneities in mantle viscosity and post-glacial rebound, Geophys. J., 98, 413-428.

Hagedoorn, J.M., Wolf, D. \& Martinec, Z., 2007. An estimate of global sealevel rise inferred from tide-gauge measurements using glacial isostatic models consistent with the relative sea-level record, Pure appl. Geophys., 164, 791-818.

Han, D. \& Wahr, J., 1989. Post-glacial rebound analysis for a rotating Earth, in Slow Deformations and Transmission of Stress in the Earth, Vol. 49, pp. 1-6, eds Cohen, S. \& Vanicek, P., AGU Monogr. Series.

James, T., 1991. Post-glacial Deformation, PhD thesis, Princeton University, Princeton, $\mathrm{NJ}$

James, T.S. \& Ivins, E.R., 1998. Predictions of Antarctic crustal motions driven by present-day ice sheet evolution and by isostatic memory of the Last Glacial Maximum, J. geophys. Res., 103, 4993-5017.

Jones, E., Oliphant, T. \& Peterson, P., 2001. SciPy: Open source scientific tools for Python, https://www.scipy.org.

Kachuck, S.B., 2017. giapy: Glacial isostatic adjustment in Python, https://github.com/skachuck/giapy .

Kaufmann, G. \& Wu, P., 1998. Lateral asthenospheric viscosity variations and postglacial rebound: a case study for the Barents Sea, Geophys. Res. Lett., 25, 1963-1966.

Kendall, R.A., Mitrovica, J.X. \& Milne, G.A., 2005. On post-glacial sea level - II. Numerical formulation and comparative results on spherically symmetric models, Geophys. J. Int., 161, 679-706.

Klemann, V. \& Martinec, Z., 2011. Contribution of glacial-isostatic adjustment to the geocenter motion, Tectonophysics, 511, 99-108.

Klemann, V., Martinec, Z. \& Ivins, E.R., 2008. Glacial isostasy and plate motions, J. Geodyn., 46, 95-103.

Konrad, H., Thoma, M., Sasgen, I., Klemann, V., Grosfeld, K., Barbi, D. \& Martinec, Z., 2014. The deformational response of a viscoelastic solid earth model coupled to a thermomechanical ice sheet model, Surv. Geophys., 35, 1441-1458.

Konrad, H., Sasgen, I., Klemann, V., Thoma, M., Grosfeld, K. \& Martinec, Z., 2016. Sensitivity of grounding-line dynamics to viscoelastic deformation of the solid-earth in an idealized scenario, Polarforschung, 85, 89-99.

Lambeck, K. \& Nakada, M., 1990. Late Pleistocene and Holocene sealevel change along the Australian coast, Palaeogeog. Palaeoclimat. Palaeoecol., 89, 143-176.

Lambeck, K., Purcell, A., Johnston, P., Nakada, M. \& Yokoyama, Y., 2003. Water-load definition in the glacio-hydro-isostatic sea-level equation, Quat. Sci. Rev., 22, 309-318.

Latychev, K., Mitrovica, J.X., Tromp, J., Tamisiea, M.E., Komatitsch, D. \& Christara, C.C., 2005. Glacial isostatic adjustment on 3-D earth models: a finite-volume formulation, Geophys. J. Int., 161, 421-444.

Martinec, Z., 2000. Spectral-finite element approach to three-dimensional viscoelastic relaxation in a spherical earth, Geophys. J. Int., 142, $117-141$.

Martinec, Z. \& Hagedoorn, J., 2005. Time-domain approach to linearized rotational response of a three-dimensional viscoelastic earth model induced by glacial isostatic adjustment: I. Inertia-tensor perturbations, Geophys. J. Int., 163, 443-462.

Martinec, Z. \& Hagedoorn, J., 2014. The rotational feedback on linearmomentum balance in glacial isostatic adjustment, Geophys. J. Int., 199, 1823-1846.

Milne, G.A. \& Mitrovica, J.X., 1998. Postglacial sea-level change on a rotating Earth, Geophys. J. Int., 133, 1-19.

Milne, G.A., Mitrovica, J.X. \& Davis, J.L., 1999. Near-field hydro-isostasy: the implementation of a revised sea-level equation, Geophys. J. Int., 139, 464-482.

Mitrovica, J.X., 1994. A spectral formalism for computing threedimensional deformations due to surface loads, J. geophys. Res., 99, 7057-7073.

Mitrovica, J.X. \& Milne, G.A., 2003. On post-glacial sea level: I. General theory, Geophys. J. Int., 154, 253-267.
Mitrovica, J.X. \& Peltier, W., 1991. On postglacial geoid subsidence over the equatorial oceans, J. geophys. Res., 96, 20 053-20 071.

Mitrovica, J.X., Gomez, N., Morrow, E., Hay, C., Latychev, K. \& Tamisiea, M.E., 2011. On the robustness of predictions of sea level fingerprints, Geophys. J. Int., 187, 729-742.

Nakada, M. \& Lambeck, K., 1987. Glacial rebound and relative sea-level variations, Geophys. J., 90, 171-224.

Peltier, W.R., 1974. The impulse response of a Maxwell Earth, Rev. Geophys. Space Phys., 12, 649-669.

Peltier, W.R., 1998. Postglacial variations in the level of the sea: implications for climate dynamics and solid-earth geophysics, Rev. Geophys., 36, 603-689.

Riva, R.E.M., Bamber, J.L., Lavalle, D.A. \& Wouters, B., 2010. Sea-level fingerprint of continental water and ice mass change from GRACE, Geophys. Res. Lett., 37, L19605, doi:10.1029/2010GL044770.

Riva, R.E.M., Sun, Y., Ditmar, P. \& Rietbroek, R., 2018, A global empirical GIA model based on GRACE data, in Geophysical Research Abstracts, Vol. 20, EGU2018-12389, EGU General Assembly.

Schotman, H.H.A., 2007. Shallow Earth rheology from glacial isostasy and satellite gravity - a sensitivity analysis for GOCE, Ph.D. thesis, Delft University of Technology, The Netherlands.

Schotman, H.H.A. \& Vermeersen, L.L.A., 2005. Sensitivity of glacial isostatic adjustment models with shallow low-viscosity earth layers to the ice-load history in relation to the performance of GOCE and GRACE, Earth planet. Sci. Lett., 236, 828-844.

Simon, K.M., James, T.S. \& Ivins, E.R., 2010. Ocean loading effects on the prediction of Antarctic glacial isostatic uplift and gravity rates, J. Geod., 84, 305-317.

Simon, K.M., James, T.S., Henton, J.A. \& Dyke, A.S., 2016. A glacial isostatic adjustment model for the central and northern Laurentide Ice Sheet based on relative sea level and GPS measurements, Geophys. J. Int., 205, 1618-1636.

Souček, O. \& Martinec, Z., 2011. ISMIP-HEINO experiment revised: effect of higher-order approximation and sensitivity study, J. Glaciol., 57, $1158-1170$.

Spada, G. \& Stocchi, P., 2007. SELEN: A Fortran 90 program for solving the 'sea-level equation', Comput. Geosci., 33, 538-562.

Spada, G. et al., 2004. Modeling Earth's post-glacial rebound, EOS, Trans. Am. geophys. Un., 85, 62-64.

Spada, G. et al., 2011. A benchmark study for glacial isostatic adjustment codes, Geophys. J. Int., 185, 106-132.

Steffen, H., Kaufmann, G. \& Wu, P., 2006. Three-dimensional finite-element modeling of the glacial isostatic adjustment in Fennoscandia, Earth Planet. Sci. Lett., 250, 358-375.

Tegmark, M., 1996. An icosahedron-based method for pixelizing the celestial sphere, Astrophys. J. Lett., 470, L81-L84.

van Dael, N.G., 2006. Sensitivity of glacial isostatic adjustment induced rotational changes to the ice-load history, Master's thesis, Delft University of Technology, the Netherlands.

van der Wal, W. \& IJpelaar, T., 2017. The effect of sediment loading in Fennoscandia and the Barents sea during the last glacial cycle on glacial isostatic adjustment observations, Solid Earth, 8, 955-968.

van der Wal, W., Wu, P., Wang, H. \& Sideris, M.G., 2010. Sea levels and uplift rate from composite rheology in glacial isostatic adjustment modeling, J. Geodyn., 50, 38-48.

Vellinga, P., Katsman, C., Sterl, A. \& Beersma, J., 2008. Onderzoek naar bovengrensscenario's voor klimaatverandering voor overstromingsbescherming van Nederland, Tech. rep., Achtergrondrapport Deltacommissie, http://www. deltacommissie, com/doc/Onderzoek, 20.

Whitaker, J.S., 2016. pyspharm: An object-oriented python interface to the NCAR SPHEREPACK library, https://github.com/jswhit/pyspharm/blob/master/html.

Wu, P., 2004. Using commercial finite element packages for the study of earth deformations, sea levels and the state of stress, Geophys. J. Int., 158, 401-408.

Wu, P. \& Peltier, W.R., 1982. Viscous gravitational relaxation, Geophys. J. R. astr. Soc., 70, 435-485. 
Wu, P. \& van der Wal, W., 2003. Postglacial sealevels on a spherical, selfgravitating viscoelastic earth: Effects of lateral viscosity variations in the upper mantle on the inference of viscosity contrasts in the lower mantle, Earth Planet. Sci. Lett., 211, 57-68.

\section{APPENDIX: CONVERGENCE OF THE VEGA SOLUTIONS}

As stated, there is no analytical solution to the forward modelling of GIA with a free boundary-value function defined by the SLE. To compare numerical results of individual numerical codes, we showed the differences between the individual solutions and a reference solution. In this section, we will demonstrate that the VEGA solution converges with increasing resolution of the SH parametrization of the GIA field variables and with a finer spatial grid for solving the SLE.

To do so, we use the setup of benchmark D and run the VEGA code for four different combinations of cut-off degree $j_{\max }$ of the SH parametrization of the GIA field variables and spatial grid $G$ for solving the SLE. We begin with a low spatial resolution and gradually increase it. Table A1 summarizes the four combinations $\mathrm{Ci}, i=1, \ldots, 4$, employed, where the VEGA solution for the Ci combination of input parameters will be denoted by $\mathrm{VEGA}^{\mathrm{Ci}}, i=1, \ldots, 4$.

Table A1. The four combinations $\mathrm{C} 1, . ., \mathrm{C} 4$ of cut-off degree $j_{\max }$ and spatial grid $G$ of $m$ Gauss-Legendre latitudinal nodes and $n$ longitudinal latitudinal nodes for solving the SLE $(G=m \times n)$ used to study the convergence of the VEGA solutions.

\begin{tabular}{lcc}
\hline Comb. & $j_{\max }$ & $\mathrm{G}$ \\
\hline C1 & 64 & $256 \times 512$ \\
C2 & 128 & $256 \times 512$ \\
C3 & 128 & $512 \times 1024$ \\
C4 & 256 & $512 \times 1024$ \\
\hline
\end{tabular}

The differences of the $\mathrm{VEGA}^{\mathrm{Ci}}$ solutions with respect to VEGA ${ }^{\text {ref }}$ along the co-latitudinal cross-sections shown in Figs 14 and 15 , which were used in benchmark D, are shown in the left-hand and right-hand panels of Fig. A1, respectively. We can see that the differences in $U$ and $N$ have predominantly a short-wavelength feature with spikes at the edge of ice cap (the left-hand panels of Fig. A1) and ocean basin (right-hand panels). The spikes are again caused by the combined effect of the Gibbs phenomenon generated at the coast of the ocean basin and the slowly convergent SH series for the ice cap. The frequency of the oscillations increases with increasing cut-off degree $j_{\text {max }}$, but the amplitudes decrease, which is caused by the damping effect of the viscoelastic structure of the earth model. The metrics of the differences of VEGA $^{\mathrm{Ci}}$ solutions with respect to VEGA ${ }^{\text {ref }}$ are summarized graphically in Fig. A2. It is evident from the figures that the differences decrease when moving from the $\mathrm{VEGA}^{\mathrm{C} 1}$ solution to the $\mathrm{VEGA}^{\mathrm{C} 4}$ solution, that is, when the spatial resolution of $U$ and $N$ is increasing and the grid $G$ for solving the SLE gets finer. Hence, the VEGA solution converges when the spatial resolution increases. More specifically, the difference in $U$ and $N$ of the $\mathrm{VEGA}^{\mathrm{C} 4}$ solution with respect to $\mathrm{VEGA}^{\text {ref }}$ reaches $0.23 \mathrm{~m}$ and $0.02 \mathrm{~m}$ with the root-mean squares of $0.06 \mathrm{~m}$ and $0.004 \mathrm{~m}$, respectively, along the cross-section shown in Fig. 14. The difference metrics are even smaller along the cross-section shown in Fig. 15. In addition, a bias of $1 \mathrm{~cm}$ in sea-surface variation $S$ shown in the bottom right-hand panel of Fig. A1 is caused by the approximation error of numerical quadrature used to compute $h_{\mathrm{UF}}(t)$. It also means the bias of $1 \mathrm{~cm}$ by the VEGA ${ }^{\text {ref }}$ solution may contribute to the bias in sea-surface variation $S$ shown in Fig. 15. 

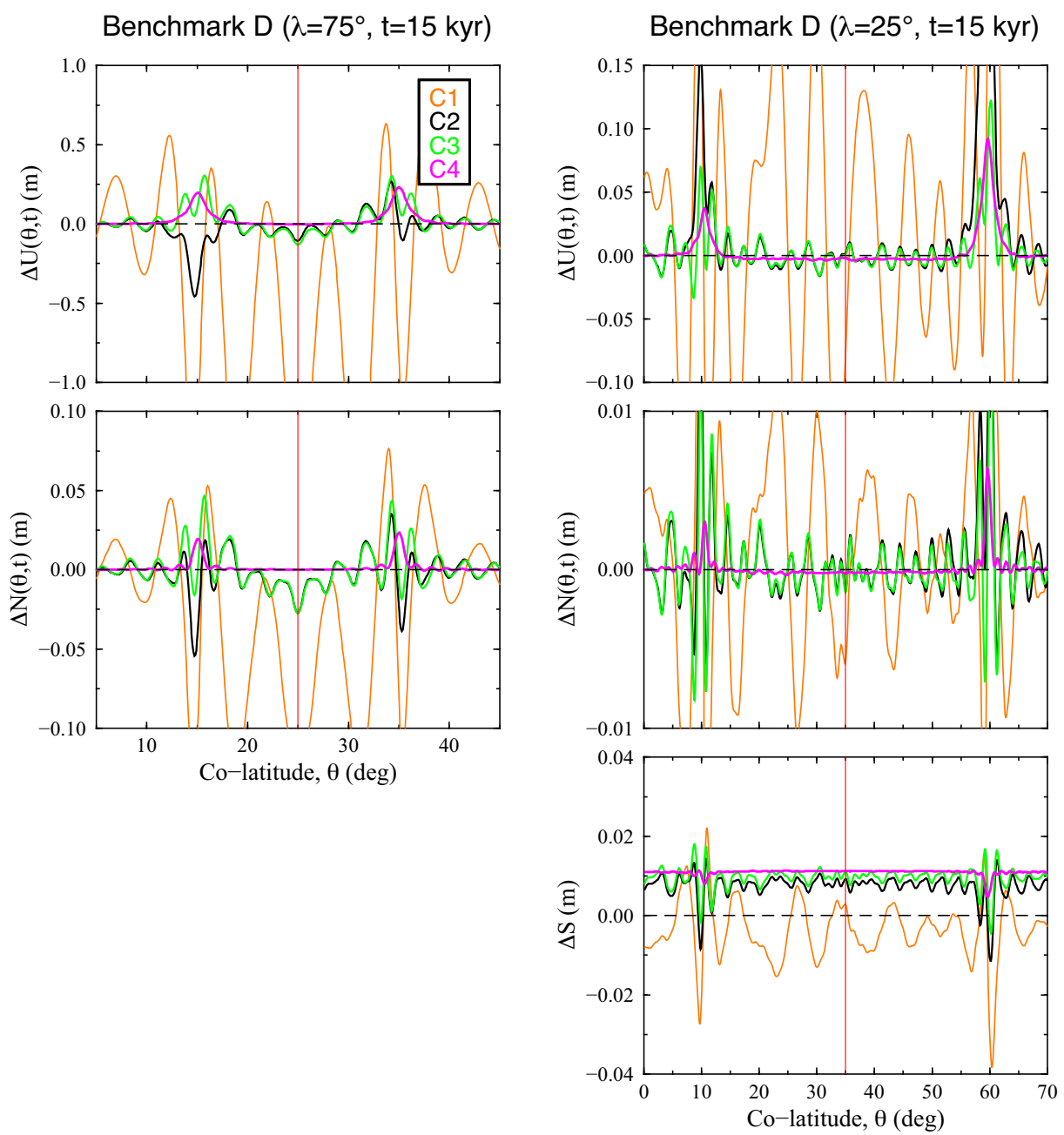

Figure A1. The differences arising from the $\mathrm{VEGA}^{\mathrm{Ci}}$ solutions, $i=1, \ldots, 4$, with respect to $\mathrm{VEGA}^{\text {ref }}$ along the co-latitudinal cross-sections shown in the right panels of Figs 14 (left) and 15 (right) for benchmark D. The four combinations C1,..,C4 of the input parameters for the $\mathrm{VEGA}^{\mathrm{Ci}}$ solutions are given in Table A1.
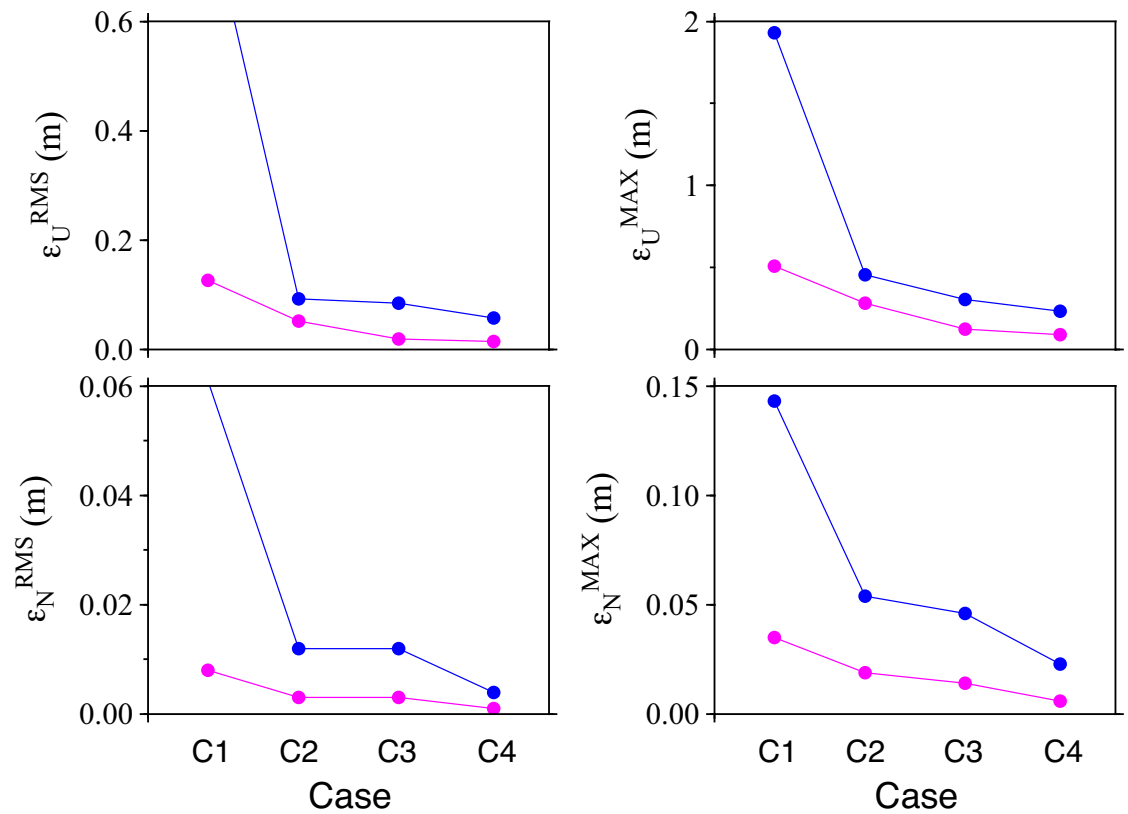

Figure A2. The root-mean squares and maximum values of the differences shown in the left-hand panels (blue) and two top right-hand panels (magenta) of Fig. A1. 